\title{
Peripheral immune tolerance alleviates the intracranial lipopolysaccharide injection- induced neuroinflammation and protects the dopaminergic neurons from neuroinflammation-related neurotoxicity
}

\author{
Yang Liu, Xin Xie, Li-Ping Xia, Hong LV, Fan Lou, Yan Ren, Zhi-Yi He and Xiao-Guang Luo* (i]
}

\begin{abstract}
Background: Neuroinflammation plays a critical role in the onset and development of neurodegeneration disorders such as Parkinson's disease. The immune activities of the central nervous system are profoundly affected by peripheral immune activities. Immune tolerance refers to the unresponsiveness of the immune system to continuous or repeated stimulation to avoid excessive inflammation and unnecessary by-stander injury in the face of continuous antigen threat. It has been proved that the immune tolerance could suppress the development of various peripheral inflammation-related diseases. However, the role of immune tolerance in neuroinflammation and neurodegenerative diseases was not clear.

Methods: Rats were injected with repeated low-dose lipopolysaccharide (LPS, $0.3 \mathrm{mg} / \mathrm{kg}$ ) intraperitoneally for 4 days to induce peripheral immune tolerance. Neuroinflammation was produced using intracranial LPS $(15 \mu \mathrm{g})$ injection. Inflammation cytokines were measured using enzyme-linked immunosorbent assay (ELISA) and quantitative real-time polymerase chain reaction (qRT-PCR). Microglial activation were measured using immunostaining of Iba-1 and ED-1. Dopaminergic neuronal damage was evaluated using immunochemistry staining and stereological counting of TH-positive neurons. Behavioral impairment was evaluated using amphetamine-induced rotational behavioral assessment.
\end{abstract}

Results: Compared with the non-immune tolerated animals, pre-treatment of peripheral immune tolerance significantly decreased the production of inflammatory cytokines, suppressed the microglial activation, and increased the number of dopaminergic neuronal survival in the substantia nigra.

Conclusions: Our results indicated that peripheral immune tolerance attenuated neuroinflammation and inhibited neuroinflammation-induced dopaminergic neuronal death.

Keywords: Microglia, Peripheral blood monocytes, Neuroinflammation, Neuroprotection, Neurodegeneration disease

\footnotetext{
* Correspondence: xgluo@cmu.edu.cn

Department of Neurology, The First Affiliated Hospital of China Medical

University, 155 North Nanjing Street, Heping District, Shenyang 110001,

People's Republic of China
} 


\section{Background}

Neuroinflammation is one of the protuberant pathological features of neurodegenerative diseases, including Parkinson's disease (PD) [1]. PD is characterized by a dramatic loss of dopaminergic (DA) neurons in the substantia nigra (SN). Characteristic features of neuroinflammation include the activation of glial cells and a series of inflammatory mediators including proinflammatory cytokines, chemokines, reactive oxygen species, and nitric oxide [2]. These elements have been proved to involve in the development of neuronal degeneration [3].

Despite the protection of the blood-brain barrier (BBB), the central nervous system (CNS) could be deeply affected by the peripheral immune. It is well established that peripheral inflammation can give rise to neuroinflammation and is linked closely to the etiology and progress of neurodegenerative diseases $[4,5]$. One famous study showed that a single intraperitoneal (i.p.) injection of lipopolysaccharide (LPS) resulted in an elevated production of proinflammatory cytokines and the delayed loss of dopaminergic neurons in the $\mathrm{SN}$ [6]. On the other hand, long-term use of non-steroidal anti-inflammatory drugs could decrease the incidence of PD [7,8]. These results suggest that the neuroinflammation can be strongly influenced by the peripheral inflammation.

Microglia, as the vital component of the brain immune system, plays a crucial role in maintaining brain microenvironment homeostasis [9]. Once activated, it can transform from resting state into activated state by changing its morphology, proliferating and initiating the inflammatory response by producing pro-inflammatory cytokines and neurotoxic mediators such as nitric oxide, prostaglandin E2, and reactive oxygen species [10] and by cross-talking with other glial cells, BBB cells, and neurons $[11,12]$. Studies have revealed that microglial over-activation caused by inflammation may disrupt the microenvironment in the brain and pose a threat to the dopaminergic neuronal survival.

Monocytes, as the promoter and organizer of the peripheral innate immune, play multiple roles in the immune system. Recently, a growing body of research is concentrated on the role of monocytes play in disorders of the CNS [13]. One of our recent studies showed that depletion of peripheral blood monocytes (PBM) attenuated not only peripheral inflammation but also neuroinflammation induced by peripheral LPS injection. Our results demonstrated that monocytes are the peripheral decisive elements in the process of neuroinflammation induced by peripheral inflammation [14]. Moreover, monocyte and microglia are considered to share the same origin of the myeloid lineage $[15,16]$; mounting evidence reveals that monocytes could infiltrate into the brain and develop into microglia [17] or construct a special group of macrophages [18]. These findings imply that the immune response of peripheral blood monocytes is of profound significance to the progression and prognosis of the neuroinflammation.

Immune tolerance refers to the refractoriness of the immune system when suffering continuous antigen threat. It is a protective mechanism of the body with suppressed peripheral immune activities in order to avoid excessive inflammatory response and unnecessary by-stander injury [19]. Endotoxic tolerance (ET) induced by a low dose of endotoxin (lipopolysaccharide, LPS) is deemed as the most essential component of immune tolerance [20]. Remarkably, LPS peripheral preconditioning has been proved to alleviate the process of several neurological disorders, such as middle cerebral artery occlusion syndrome (MCAO) [21], brain trauma [22], and brain injury [23]. These results demonstrate the important role immune tolerance plays in neuroprotection.

Immune tolerance can inhibit peripheral immune activity and reduce subsequent inflammation; however, little is known about its role in neurodegenerative diseases. Here, we proposed that the pre-treatment to induce peripheral immune tolerance can inhibit intracranial LPS injection-induced neuroinflammation and neurodegeneration. These results may provide a novel therapeutic strategy for treating neuroinflammationrelated neurodegenerative diseases.

\section{Methods \\ Animals}

Experiments were conducted on 112 male SpragueDawley rats (8 weeks old, 220-240 g, Animal Center of China Medical University), according to the National Institute of Health Guidelines (National Institutes of Health, Bethesda, USA) and approved by the ethics committee of China Medical University. Animals had unrestricted access to food and water and were housed in groups (4 rats per cage) in a temperature-controlled environment with an ambient temperature of $22{ }^{\circ} \mathrm{C}\left( \pm 1^{\circ}\right.$ C) and a 12-h light-dark cycle.

\section{Experimental procedures and grouping}

Before treatment, animals were numbered and acclimated for 1 week. Afterwards, rats were randomly divided into the following groups according to a random number table. Experimental procedures and grouping was shown as Fig. 1. In part I, three groups were involved: (1) a control group without treatment (control group, $n=15$ ), (2) a group treated with repeated intraperitoneal (i.p.) injections of phosphate buffered saline (PBS) for 4 days [PBS (i.p.) group, $n=$ 6], and (3) a group treated with repeated i.p. injections of $0.3 \mathrm{mg} / \mathrm{kg}$ LPS for 4 days [LPS (i.p.) group, $n=42$ ]. In part II, four groups were involved: (1) a 


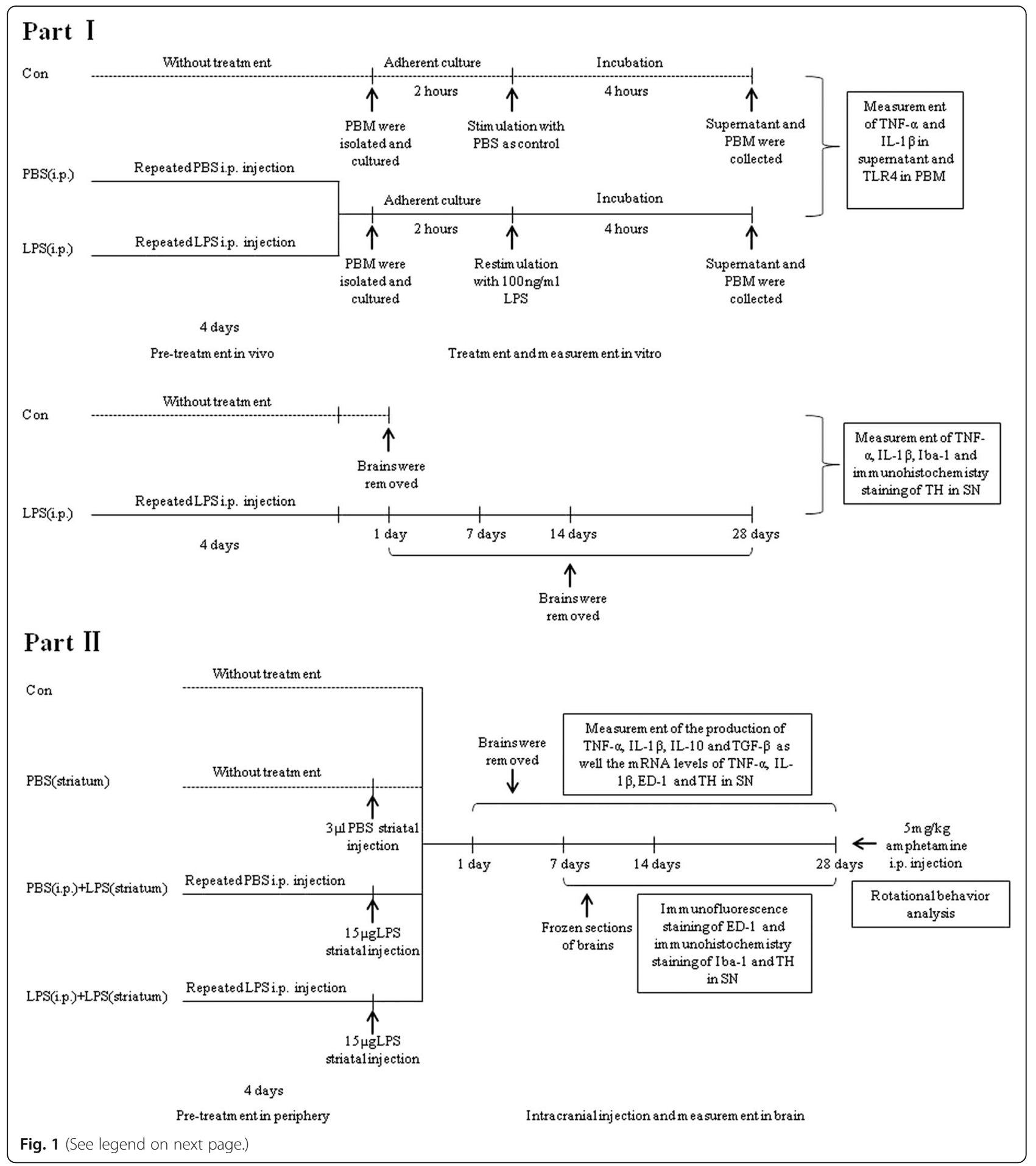

control group without treatment (control group, $n=$ 16), (2) a sham group treated with intracranial PBS injection into the right striatum [PBS (striatum) group, $n=16]$, (3) a group treated with repeated i.p. injections of PBS for 4 days plus an intracranial LPS injection into the right striatum [PBS (i.p.) + LPS (striatum) group, $n=16]$, and (4) a group treated with repeated i.p. injections of LPS for 4 days plus an intracranial LPS injection into the right striatum [LPS (i.p.) + LPS (striatum) group, $n=16$ ]

Induction and verification of peripheral immune tolerance LPS (strain O111; B4, Sigma, St Louis, USA) was dissolved in PBS ( $\mathrm{pH} 7.4)$ at a concentration of $0.1 \mathrm{mg} / \mathrm{ml}$. 


\section{(See figure on previous page.)}

Fig. 1 Study procedure and grouping. In the first part of the experiment, we confirmed that repeated low-dose LPS i.p. injection could induce immune tolerance of PBM without inflammation or dopaminergic neuronal loss in the brain. To induct endotoxic tolerance, rats were pre-treated with repeated $0.3 \mathrm{mg} / \mathrm{kg}$ LPS intraperitoneal injection for 4 days. Then, PBM were isolated, cultured, and restimulated by a hige-dose LPS. Moreover, we verified that peripheral repeated low-dose LPS intraperitoneal administration was failing to cause inflammation or dopaminergic neuronal loss in the brain parenchyma of rats. According to the different preconditioning, three groups were involved: the control group ( $n=15)$, the PBS (i.p.) group $(n=6)$, and the LPS (i.p.) group $(n=42)$. In the second part of the experiment, we investigated the neuroprotective role of peripheral immune tolerance in intracranial LPS injection-induced neuroinflammation-related neurodegeneration. Neuroinflammation-induced PD rat model was conducted by $15 \mu \mathrm{g}$ LPS intracranial injection into the right striatum. Inflammatory cytokines, microglial activation, loss of dopaminergic neurons, as well the behavior impairment were detected at indicated time points. Four groups were involved, including a control group without treatment, a PBS(striatum) group with striatal PBS injection as the sham group, a PBS (i.p.) + LPS (striatum) group with peripheral PBS pretreatment prior to striatal $15 \mu \mathrm{g}$ LPS injection, and a LPS (i.p.) + LPS (striatum) group with peripheral LPS pre-treatment prior to striatal $15 \mu \mathrm{g}$ LPS injection

To generate peripheral immune tolerance, rats were given pre-treatment with repeated i.p. injections of LPS $(0.3 \mathrm{mg} / \mathrm{kg}$ body weight/single/day) for 4 days. (A variety of concentrations have been explored in the preliminary experiment; afterwards, the dose of LPS at $0.3 \mathrm{mg} / \mathrm{kg}$ was determined ultimately. Additional file 1: Figure S1.) After the peripheral pre-treatment, rats were sacrificed for peripheral blood, and then, peripheral blood monocytes (PBM) were cultured with combined density gradient centrifugation and attachment culture method. $1 \times$ $10^{6}$ cells/ml was regarded as the appropriate cell density for incubation at $37{ }^{\circ} \mathrm{C}$ under $5 \% \mathrm{CO}_{2}$. After the adherent cell growth, medium was changed, and $100 \mathrm{ng} / \mathrm{ml}$ LPS was given to PBM in vitro for $4 \mathrm{~h}$ in order to mimic subsequent inflammation. To test the immunological competence of PBM, the levels of pro-inflammatory cytokines (TNF- $\alpha$ and IL-1 $\beta$ ) in the supernatant were measured by enzyme-linked immunosorbent assay (ELISA), while also the expression of toll-like receptor 4 (TLR4) in monocytes was measured by Western blot.

\section{Stereotaxic surgery}

For stereotaxic surgery, rats were anesthetized with an i.p. injection of chloral hydrate $(400 \mathrm{mg} / \mathrm{kg})$. Afterwards, they were placed in a stereotaxic apparatus. The skull was exposed, and a dental drill was used to make a small hole to allow for the striatal injection. LPS $(15 \mu \mathrm{g}$ in $3 \mathrm{ul}$, dissolved in PBS) was injected unilaterally at the following coordinates: AP, $-0.4 \mathrm{~mm} ; L,+3.0 \mathrm{~mm}$;,$5.5 \mathrm{~mm}$, which corresponded to the largest segment of striatum in the coronal plane [24]. (In our preliminary experiments, we explored LPS-induced PD rats model with various stereotaxic injection sites and various doses of LPS; ultimately, unilateral striatal injection of $15 \mu \mathrm{g}$ LPS was adopted as the PD model in our present study. Additional file 2: Figure S2.) The sham group was injected with vehicle (PBS, $3 \mu \mathrm{l})$. The injection was controlled at a rate of $1 \mathrm{ul} / \mathrm{min}$, and the Hamilton syringe needle was left in place for $5 \mathrm{~min}$ and then slowly withdrawn to prevent reflux of the solution.

\section{Measurement of cytokines Supernatant of cultured PBM}

At $4 \mathrm{~h}$ from $100 \mathrm{ng}$ LPS treatment, supernatant was collected and the amount of TNF- $\alpha$ and IL-1 $\beta$ was measured with ELISA techniques. ELISA was performed according to manufacturer's instructions (R\&D Company, Minneapolis, USA). The detection limits of TNF- $\alpha$ and IL-1 $\beta$ were 800 and $50 \mathrm{pg} / \mathrm{ml}$.

\section{$S N$ of rat brain}

Rats were sacrificed 1, 7, 14, and 28 days after the final treatment. The right $\mathrm{SN}$ were dissected out on ice. The amount of pro-inflammation cytokines (TNF- $\alpha$, IL-1 $\beta$ ) and anti-inflammation cytokines (TGF- $\beta$, IL-10) from SN was measured with ELISA techniques. Tissues were homogenized in $1000 \mathrm{ul}$ of ice-cold PBS (0.01M, pH 7.4) and centrifuged at $12,000 \mathrm{~g}$ at $4{ }^{\circ} \mathrm{C}$ for $15 \mathrm{~min}$. Centrifugal supernatant was collected, and ELISA was then performed according to manufacturer's instructions (R\&D Company, Minneapolis, USA). The detection limit of TNF- $\alpha$, IL- $1 \beta$, IL-10, and TGF- $\beta$ were $300,80,50$, and $200 \mathrm{pg} / \mathrm{ml}$, respectively.

\section{Western blot analysis Cultured PBM}

At $4 \mathrm{~h}$ from $100 \mathrm{ng}$ LPS treatment, cultured PBM was scraped and collected with $150 \mathrm{ul}$ of ice-cold lysis buffer ( $1 \mathrm{ml}$ RIPA Lysis Buffer, $10 \mu \mathrm{l}$ PMSF, Biyotime, Shanghai, China) for $30 \mathrm{~min}$ splitting and then centrifuged $\left(12,000 \mathrm{~g}\right.$ at $4{ }^{\circ} \mathrm{C}$ for $\left.15 \mathrm{~min}\right)$. Centrifugal supernatant was kept at $-80{ }^{\circ} \mathrm{C}$ until used for analysis.

\section{$S N$ of rat brain}

Rats were sacrificed $1,7,14$, and 28 days after the final treatment, and the right $\mathrm{SN}$ were dissected out on ice. Tissues were homogenized in $1000 \mathrm{ul}$ of ice-cold lysis buffer (1 ml RIPA Lysis Buffer, $10 \mu$ l PMSF, Biyotime, Shanghai, China) and lysed at $4{ }^{\circ} \mathrm{C}$ for $30 \mathrm{~min}$. The resultant lysates were centrifuged $\left(12,000 \mathrm{~g}\right.$ at $4{ }^{\circ} \mathrm{C}$ for 
$30 \mathrm{~min}$ ) to remove particulate materials. Centrifugal supernatant was kept at $-80{ }^{\circ} \mathrm{C}$ until used for analysis.

Afterwards, protein concentration was determined using the BCA kit (Biyotime, Shanghai, China) and enzyme analyzer. Equal amounts of proteins $(15 \mu \mathrm{g}$ for cell lysates; $30 \mu \mathrm{g}$ for tissue lysates) were separated by $10 \%$ SDS-PAGE gels and transferred to polyvinylidene difluoride (PVDF) membranes (Bio-Rad, California, USA) using an electrophoretic transfer system (Bio-Rad, California, USA), which were blocked in 5\% fat-free milk (room temperature, $2 \mathrm{~h}$ ). The membranes were incubated in primary antibodies (mouse antibody against TLR4, Abcam, Danvers, USA, 1:400; goat antibody against Iba-1, Wako, Tokyo, USA, 1:800; mouse antibody against GAPDH, Abcam, Danvers, USA, 1:1000) overnight at $4{ }^{\circ} \mathrm{C}$. Then, the membranes were rinsed with tris-buffered saline containing $0.1 \%$ tween 20 (TBST) 3 times for $10 \mathrm{~min}$ per time before incubation in secondary antibody (horseradish peroxidase-labeled anti-mouse IgG, Abcam, Danvers, USA, 1:2000; horseradish peroxidase-labeled anti-goat IgG, Abcam, Danvers, USA, 1:2000) for $2 \mathrm{~h}$ at room temperature. This was followed by treatment with the ECL chemiluminescent reagents (Bio-Rad, California, USA) and exposed using the ECL chemiluminescence detector (Bio-Rad, California, USA). A density measurement for each band was performed with the ImageJ software. The relative levels of the TLR4 and Iba-1 were normalized against the levels of GAPDH and expressed graphically.

\section{Quantitative real-time PCR}

Rats were sacrificed, and the right $\mathrm{SN}$ was immediately isolated. The total RNA from the right $\mathrm{SN}$ was extracted using Trizol (Takara, Dalian, China) according to manufacturer's instructions and was then quantified photometrically. Reverse transcription was performed with the PrimeScript ${ }^{\text {tw }}$ RT Reagent Kit (Takara, Dalian, China). Quantitative real-time PCR (qRT-PCR) was conducted using the SYBR Premix Ex Taq ${ }^{\text {Tix }}$ (Takara, Dalian, China) and a Light Cycler 480 II Real-Time PCR system (Roche Diagnostics, Basel, Switzerland). The relative expression among the groups was calculated using the 2 $-\Delta \Delta \mathrm{Ct}$ method. The primer sequences were listed as the following: rat GAPDH forward, GCAGCCCA GAACATCATCC reverse, GTCATCATACTTGGCAG GTT; rat TNF- $\alpha$ forward, ACTGAACTTCGGGGTG ATTG reverse, GTGGGTGAGGAGCACGTAGT; rat IL-1 $\beta$ forward, AGCTGCACTGCAGGCTTCGAGATG reverse, GAACTGTGCAGACTCAAACTCCAC; rat ED-1 forward, TGAGGTTCCCTGTGTGTCTG reverse, GGTTGTAGGTGTCTCCGTGAA; rat TH forward, CTACTGTCCGCCCGTGATTT reverse, CACA GGCTGGTAGGTTTGATC.

\section{Tissue preparation}

At $1,7,14$, and 28 days post the striatal injection, the rats were deeply anesthetized and perfused through the aorta with $0.9 \%$ saline, followed by $4 \%$ paraformaldehyde dissolved in PBS (0.01M, pH 7.4). Brains were dissected and fixed in $4 \%$ buffered paraformaldehyde and cryoprotected in $30 \%$ sucrose solution at $4{ }^{\circ} \mathrm{C}$ for 2 days. Coronal serial sections (20 um) throughout the $\mathrm{SN}$ were cut using a freezing microtome (CM1900, Leica, Germany) maintained at $-20{ }^{\circ} \mathrm{C}$. Afterwards, the sections were stored in $65 \%$ glycerinum at $-20{ }^{\circ} \mathrm{C}$ for histological analysis.

\section{Immunohistochemistry and immunofluorescence}

Brain sections were rinsed with PBS and pre-treated with $0.3 \% \mathrm{H}_{2} \mathrm{O}_{2}$ for $15 \mathrm{~min}$ to inhibit endogenous peroxidase activity, and then rinsed in PBS 3 times for $5 \mathrm{~min}$ per time. Then, the sections were blocked with $0.5 \%$ bovine serum albumin (BSA) for $30 \mathrm{~min}$ at room temperature. Sections were incubated overnight at $4{ }^{\circ} \mathrm{C}$ with the following primary antibodies: mouse antibody against ED1 (CD68, Abcam, Danvers, USA, 1:400), goat antibody against tyrosine hydroxylase (TH, Abcam, Danvers, USA, 1:800), goat antibody against Iba-1 (Wako, Tokyo, USA, 1:800). The sections were washed with PBS post to the incubation. For fluorescence staining, sections were incubated with Alexa Fluor 594-conjugated secondary antibodies (Molecular Probes, California, USA, 1:1000) for $2 \mathrm{~h}$ at room temperature. Besides, Hoechst (Biyotime, Shanghai, China) was used to label the cell nucleus. Immunostaining sections were evaluated using a fluorescence microscope (Olympus BX51, Tokyo, Japan). For histochemistry staining, sections were incubated with a biotinylated rabbit-anti-goat secondary antibody for $40 \mathrm{~min}$ after the incubation of primary antibodies, followed by incubation with avidin-conjugated horseradish peroxidase for $40 \mathrm{~min}$. DAB was used to develop the color. The sections were dehydrated using an alcohol gradient, cleared in xylene, and cover slipped with neutral gum for microscopic analysis.

\section{Stereological counting of dopaminergic neurons}

To evaluate the loss of dopaminergic neurons, the unbiased stereological counting of the TH-positive neurons was made using the optical fractionator method performed on an Olympus Computer Assisted Stereological Toolbox system, version 2.1.4 (Olympus, Ballerup, Denmark). This sampling technique was previously described in detail with some modifications $[25,26]$, which is unaffected by the volume of reference or the size of the counted elements [27]. The coronal serial sections $(20 \mu \mathrm{m})$ used for counting covered the entire SN, from the rostral tip of the pars compacta (AP, $-4.30 \mathrm{~mm}$ ) back to the caudal end of the pars reticulate (AP, - 
$6.72 \mathrm{~mm}$ ). This generally yielded about 120 sections in each rat. For the estimation of the total numbers of THpositive neurons in the $\mathrm{SN}$, every sixth section was counted; moreover, a counting frame of $40 \times 40 \mu \mathrm{m}$ and a grid size of $150 \times 150 \mu \mathrm{m}$ was used. Guard volume ( $4 \mu \mathrm{m}$ from the top, 4-6 $\mu \mathrm{m}$ from the bottom of the section) was excluded from both surfaces to avoid the problem of lost cap, and only the profiles that came into focus within the counting volume (with a depth of $10 \mu \mathrm{m})$ were counted. The estimate of the total numbers of TH-positive neurons was calculated according to the optical fractionator formula [27].

\section{Behavioral assessment}

At 28 days, test for amphetamine-induced rotational behavior was carried out. For the test, rats were placed in a hemispherical bowl immediately after receiving $5 \mathrm{mg} / \mathrm{kg}$ amphetamine (dissolved in normal saline, Sigma, St Louis, USA) i.p. injections. The number of ipsilateral $360^{\circ}$ turns was measured every minute for 180 min per rat.

\section{Statistical analysis}

Data are presented as the mean \pm standard error of the mean (SEM). Tests of variance homogeneity, normality, and distribution were performed to ensure that the assumptions required for standard parametric analysis of variance were satisfied. When the homogeneity of variance was equal, one-way analysis of variance (ANOVA) followed by Least Significant Digit (LSD) test was used. When the assumption of homogeneity of variances is not met, Welch's test was applied and Dunnett T3 test was used for comparison of pairwise significance. All data were analyzed using SPSS 19.0, and $P$ values less than 0.05 were considered statistically significant.

\section{Results}

\section{Repeated low-dose LPS intraperitoneal injection-induced} endotoxic tolerance of PBM

To test whether endotoxic tolerance could be induced by repeated low-dose LPS intraperitoneal injection, TNF- $\alpha$ and IL- $1 \beta$ secreted by PBM and TLR4 expressed in PBM were quantified post to a single-dose LPS (100 ng/ml) administration. In the PBS (i.p.) group, which received repeated intraperitoneal injection of PBS prior to a single high dose of LPS administration, the TNF- $\alpha$ and IL- $1 \beta$ amounts in the supernatant were both increased; moreover, the expression of TLR4 was also upregulated, compared with the control group (without LPS restimulation), as shown in Fig. 2. Compared to the PBS (i.p.) group, a significant decrease in the levels of TNF- $\alpha(10.91 \%, P<0.01)$ and IL-1 $\beta(5.04 \%, P<0.05)$ were observed in the LPS (i.p.) group, which was caused by repeated $0.3 \mathrm{mg} / \mathrm{kg}$ LPS intraperitoneal injection. Besides, the TLR4 expression was upregulated by $25.71 \%$ $(P<0.01)$ in the LPS (i.p.) group than in the control

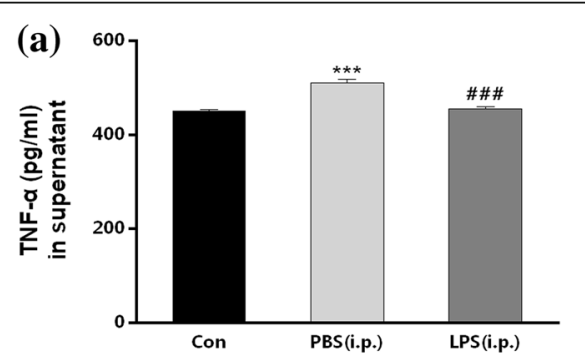

(c)

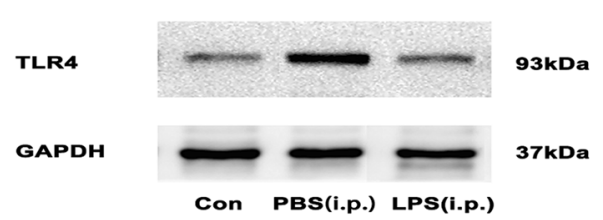

- Con

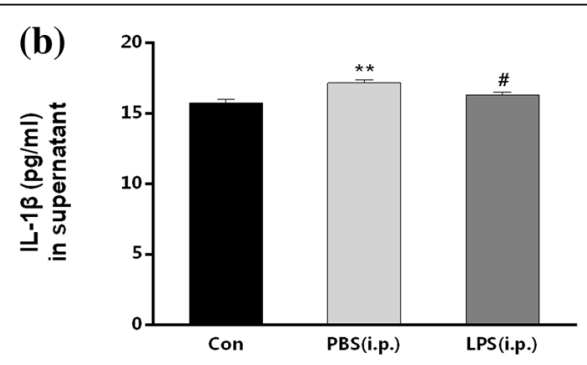

(d)

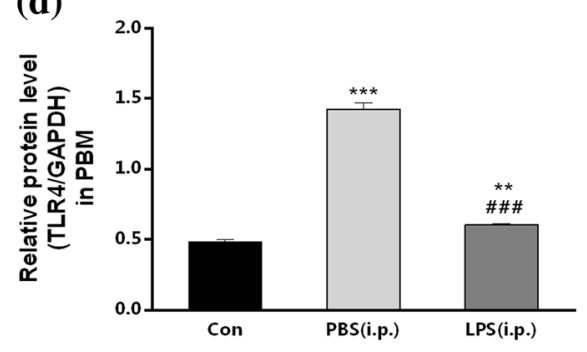

Fig. 2 Repeated low-dose LPS intraperitoneal injection-induced endotoxic tolerance of PBM. PBM from three groups with or without LPS pretreatment in vivo were isolated and cultured. Then, TNF- $\alpha$ and IL-1 $\beta$ secreted by PBM and TLR4 expressed in PBM were quantified post to a single high-dose LPS $(100 \mathrm{ng} / \mathrm{ml})$ administration for $4 \mathrm{~h}$ in vitro. The productions of TNF- $\alpha$ and IL-1 $\beta$ were decreased and the expression of TLR4 in PBM was downregulated after LPS restimulation by repeated $0.3 \mathrm{mg} / \mathrm{kg}$ LPS intraperitoneal injection in LPS (i.p.) group. $\mathbf{a}, \mathbf{b}$ The levels of TNF-a (a) and IL-1 $\beta$ (b) in the supernatant were detected by ELISA. $\mathbf{c}$, d The expression of TLR4 in PBM was quantified by measuring band intensities using ImageJ software. The values were normalized to GAPDH. ${ }^{* *} P<0.01,{ }^{* * *} P<0.001$ vs. control group. $\# P<0.05, \# \# \#>0.001$ vs. PBS (i.p.) group. The data are presented as the mean $\pm \operatorname{SEM}(n=6)$ 
group, but it was still downregulated by $57.57 \%(P<$ 0.01 ), compared with the PBS (i.p.) group. These results demonstrated that endotoxic tolerance of PBM could be induced by repeated low-dose LPS intraperitoneal injection in rats.

\section{Repeated low-dose LPS intraperitoneal injection was not} sufficient to cause inflammation in the SN

To verify that the pre-treatment with repeated low-dose LPS intraperitoneal injection was not sufficient to cause inflammation in the $\mathrm{SN}$, we investigated the levels of pre-inflammatory cytokines (TNF- $\alpha$ and IL-1 $\beta$ ) and the expression of Iba- 1 in the right SN of rats from the LPS (i.p.) group at 1, 7, 14, and 28 days post to the last peripheral LPS injection. As showed in Fig. 3, there was no significant difference between the control group and any other time points of the LPS (i.p.) group, neither in the amounts of TNF- $\alpha$ (Fig. 3a), IL-1 $\beta$ (Fig. 3b) nor in the expression of Iba-1 (Fig. 3c, d). Time-dependent effect did not exist either. The results indicated that repeated low-dose LPS intraperitoneal injection was not sufficient to cause inflammation in the $\mathrm{SN}$ of rats.

\section{Repeated low-dose LPS intraperitoneal injection was not} sufficient to cause dopaminergic neuronal loss in the SN To confirm that the pre-treatment with repeated lowdose LPS intraperitoneal injection was not sufficient to cause dopaminergic neuronal loss in the $\mathrm{SN}$, we carried out immunohistochemical staining of $\mathrm{TH}$ and stereological counting of TH-positive neurons in the nonintracranial injected SN of rats from the LPS (i.p.) group at $1,7,14$, and 28 days post to the last peripheral LPS injection. As showed in Fig. 4, dopaminergic neuronal morphology (Fig. 4a) and the total numbers of $\mathrm{TH}$ positive cells (Fig. 4b) expressed no significant difference between the control group and any other time points of the LPS (i.p.) group. The results indicated that repeated low-dose LPS intraperitoneal injection was not sufficient to cause dopaminergic neuronal loss in the $\mathrm{SN}$ of rats.

\section{Peripheral immune tolerance preconditioning decreased intracranial LPS injection-induced pro-inflammatory cytokines in the SN}

To test whether peripheral immune tolerance preconditioning was able to prevent intracranial LPS injection-induced neuroinflammation, we first investigated the expressions of pro-inflammatory cytokines (TNF- $\alpha$ and IL-1 $\beta$ ) in the right SN by ELISA. Striatal LPS administration caused a time-dependent increase of TNF- $\alpha$ and IL-1 $\beta$ expressions in the SN both in the groups with or without peripheral LPS preconditioning (Fig. 5). Compared with the PBS (i.p.) + LPS (striatum) group, the endotoxic tolerant pre-treatment significantly decreased the expression of TNF- $\alpha$ by
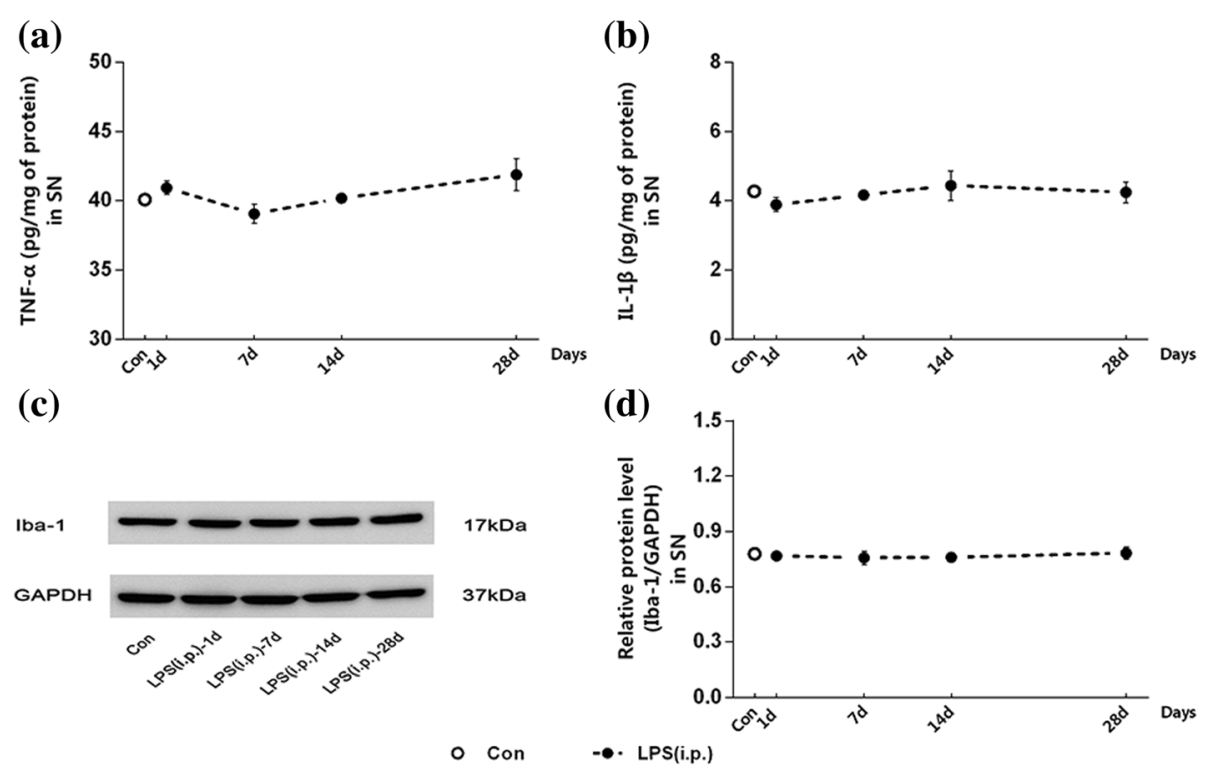

Fig. 3 Repeated low-dose LPS intraperitoneal injection preconditioning did not cause inflammation in the SN. We investigated the levels of proinflammatory cytokines and the expression of Iba-1 in the right SN of rats at 1, 7, 14, and 28 days post to the peripheral LPS injection. There was no significant difference between the control group and any other time points of the LPS (i.p.) group in the amounts of TNF- $a$ and IL-1 $\beta$ nor in the expression of Iba-1. $\mathbf{a}, \mathbf{b}$ The levels of TNF- $\mathbf{a}(\mathbf{a})$ and IL-1 $\beta(\mathbf{b})$ in the right SN were detected by ELISA. $\mathbf{c}$, $\mathbf{d}$ The expression of Iba-1 in the right SN was quantified by measuring band intensities using ImageJ software. The values were normalized to GAPDH. The data are presented as the mean $\pm \operatorname{SEM}(n=6)$ 


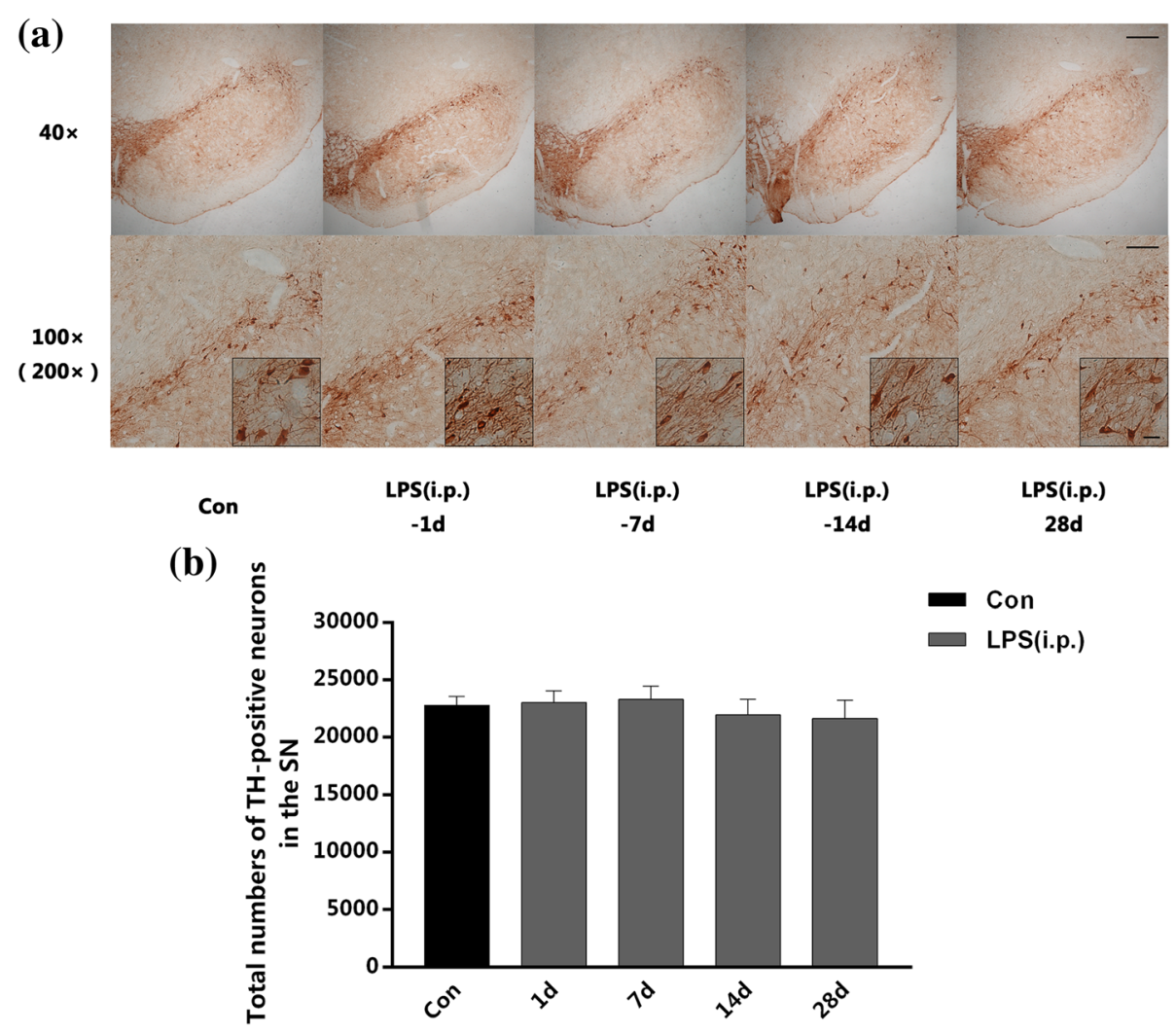

Fig. 4 . Repeated low-dose LPS intraperitoneal injection preconditioning did not cause dopaminergic neuronal loss in the SN. We carried out immunohistochemical staining of TH and stereological counting of TH-positive neurons in the non-intracranial injected SN of rats at 1, 7, 14, and 28 days after the peripheral LPS injection. There was no significant differences between the control group and any other time points of the LPS (i.p.) group in the total numbers of TH-positive cells. a Immunohistochemistry staining on frozen sections of TH in the control group and any other time points of the LPS (i.p.) group. $\mathbf{b}$ Total numbers of TH-positive neurons in the right SN collected by stereological counting $(n=3$, each group). The data are presented as the mean \pm SEM. Scale bars, $250 \mu \mathrm{m}(40 \times), 100 \mu \mathrm{m}(100 \times)$, and $25 \mu \mathrm{m}(200 \times)$

12.86, 10.45, and 7.35\% (Fig. 5a-c), as well as decreased the expression of IL-1 $\beta$ by $23.84,19.68$, and $21.42 \%$ at 1,7 , and 14 days individually post to the striatal LPS injection (Fig. 5d-f).

Then, we examined the expression profiles of TNF$\alpha$ and IL-1 $\beta$ in the right SN by qRT-PCR. Significant decreases in the mRNA levels of TNF- $\alpha$ and IL-1 $\beta$ were observed in the LPS (i.p.) + LPS (striatum) group compared to the PBS (i.p.) + LPS (striatum) group at indicated time points (Fig. 6). The LPS (i.p.) + LPS (striatum) group showed significant decreased TNF- $\alpha$ mRNA levels at 1,7 , and 14 days (Fig. $6 a-c$ ), as well as decreased IL-1 $\beta$ mRNA levels at 1 and 7 days (Fig. 6d-f) individually post to the striatal LPS injection, compared with the PBS (i.p.) + LPS (striatum) group. Striatal vehicle injection [PBS (i.p.) group] made no differences in the amounts of pro-inflammatory cytokines compared with the control group.

The results showed that peripheral immune tolerance preconditioning decreased intracranial LPS injectioninduced pro-inflammatory cytokines in the $\mathrm{SN}$.
Peripheral immune tolerance preconditioning did not alter intracranial LPS injection-induced anti-inflammatory cytokines in the SN

To test whether peripheral immune tolerance preconditioning was able to prevent intracranial LPS injectioninduced increase of anti-inflammatory factors, we measured the expressions of anti-inflammatory cytokines (IL-10 and TGF- $\beta$ ) in the right SN by ELISA. The IL-10 level increased and lasted at least 14 days after the striatal LPS injection in both groups with or without peripheral LPS preconditioning (Fig. 7a-c). However, the endotoxic tolerance pre-treatment only made significant increase of IL-10 by $9.54 \%$ at 7 days post to the striatal LPS exposure (Fig. 7b).

Furthermore, there were no significant difference in the expression of TGF- $\beta$ between the LPS (i.p.) + LPS (striatum) group and the PBS (i.p.) + LPS (striatum) group at any time points of our observation (Fig. $7 d-f$ ). Striatal vehicle injection [PBS (i.p.) group] made no differences in the amounts of anti-inflammatory cytokines compared with the control group. 


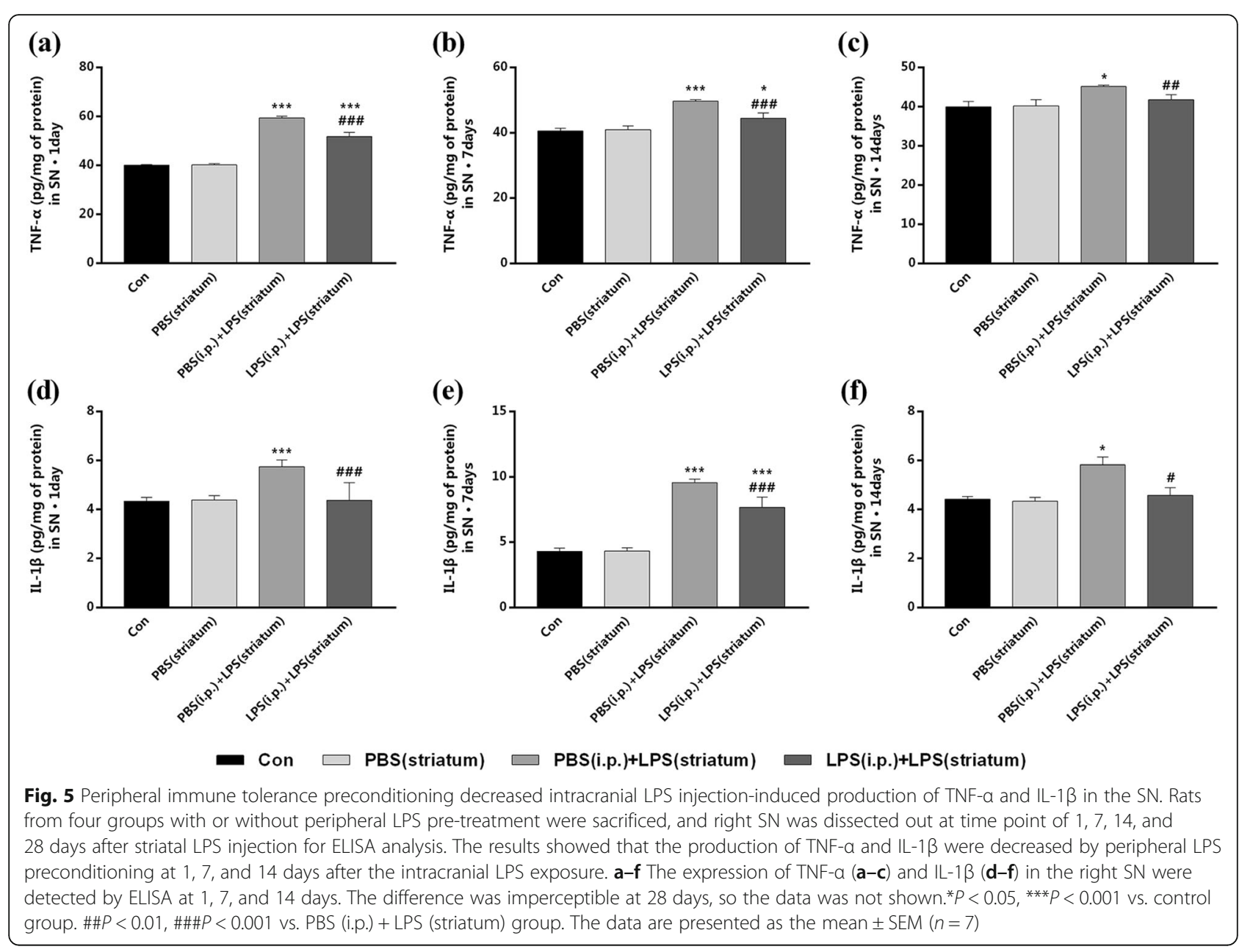

These results suggested that peripheral immune tolerance preconditioning did not alter intracranial LPS injection-induced anti-inflammatory cytokines in the $\mathrm{SN}$.

\section{Peripheral immune tolerance preconditioning inhibited intracranial LPS injection-induced microglial activation in the SN}

Firstly, we observed morphological changes of microglia using immumohistochemical staining of Iba-1. As showed in Fig. 8, microglia in the control group were "resting form" characterized by small somas, thin processes, as well as a small cellular counting (Fig. 8a). At 7 days post to the LPS exposure, amoeboid microglia had been observed in right SN (Fig. 8c, d). While microglia were "typical activated form" characterized by enlarged somas and thickened processes at 14 days post to the LPS administration (Fig. 8e, f). However, endotoxic tolerant preconditioning still resulted in fewer microglial counting and lighter immunohistochemical staining (Fig. 8d-h). Microglia in the LPS (i.p.) + LPS (striatum) group had almost returned to resting state at 28 days
(Fig. 8h), while some of microglia in the PBS (i.p.) + LPS (striatum) group had still showed deeper immunohistochemical staining (Fig. 8g), compared with the control group. Striatal vehicle injection [PBS (i.p.) group] did not alter microglial morphology (Fig. 8b), compared with the control group.

Nextly, we measured the expression of ED-1 in the SN by immunofluorescence assay.

As shown in Fig. 9, compared with control group, both two groups received intracranial LPS injection showed a time-dependent enhancement of fluorescent intensity post to the striatal LPS exposure. Compared with the PBS (i.p.) + LPS (striatum) group, the pre-treatment with endotoxic tolerance decreased the fluorescent intensity by $11.65 \%$ (Fig. 9b). A decreased fluorescent intensity of ED-1 was presented in both two groups at 14 days; furthermore, the peripheral LPS pre-treatment reduced the fluorescent intensity by $15.41 \%$ (Fig. 9c). Moreover, the fluorescence intensity of ED-1 in the LPS (i.p.) + LPS (striatum) group mainly returned to the normal state at 28 days, while it was still 1.41 times higher in the PBS (i.p.) + LPS (striatum) group than in the control group. 


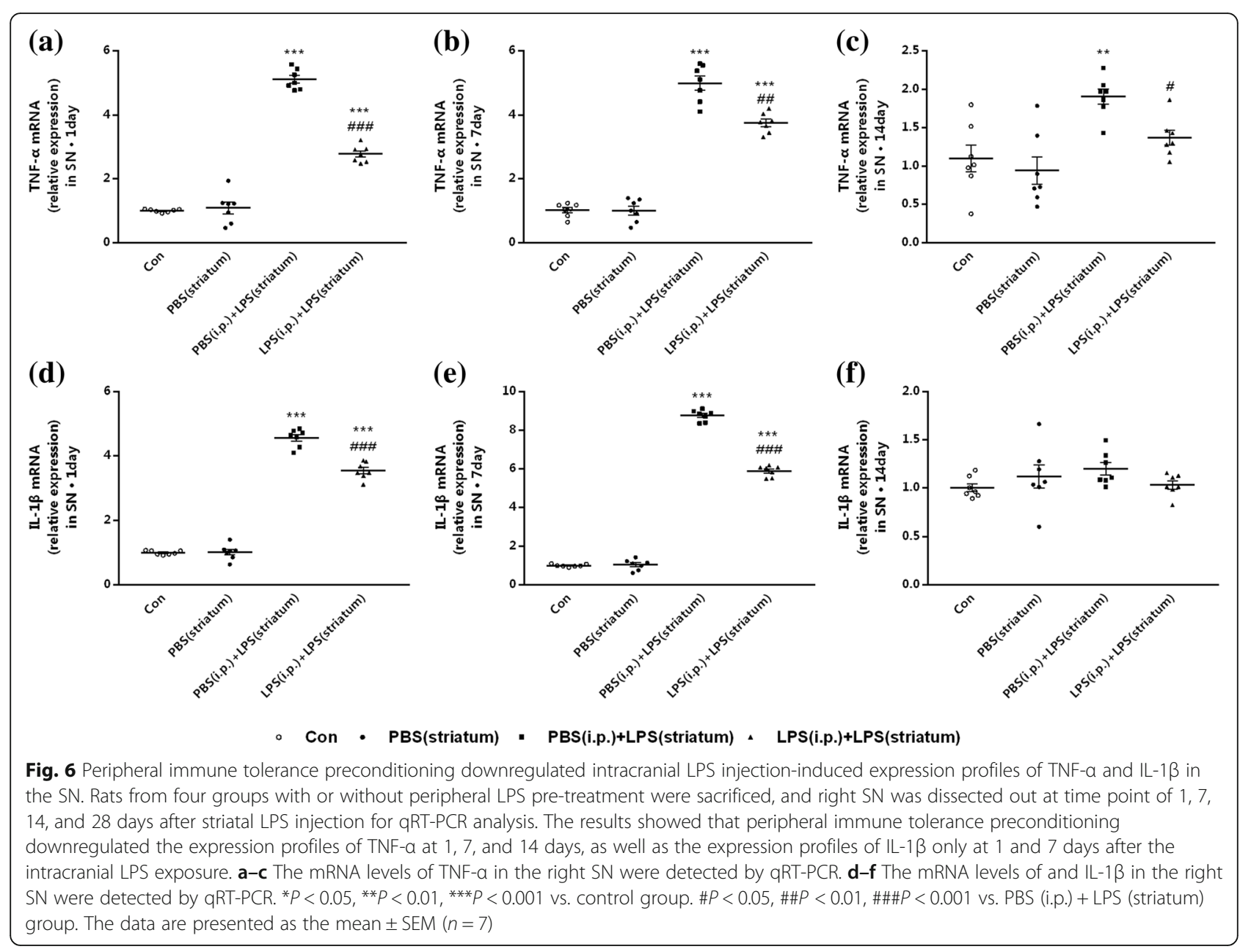

Then, the expression profiles of ED-1 in the right SN were detected by qRT-PCR. The ED-1 mRNA level showed a time-dependent increase in both two groups received intracranial LPS injection, with a maximum at 7 days and a minimum at 28 days post to the striatal LPS administration (Fig. 10a-c). In addition, pretreatment with endotoxic tolerance decreased the ED-1 mRNA level at 7 days $(P<0.01)$ (Fig. 10a) and 14 days $(P<0.01)$ (Fig. 10b), but did not significantly alter the ED-1 expression profiles at 28 days (Fig. 10c). Striatal vehicle injection [PBS (i.p.) group] made no differences in the ED-1 expression compared with the control group.

The results showed that endotoxin tolerance preconditioning inhibited intracranial LPS injection-induced microglial activation in the $\mathrm{SN}$.

Peripheral immune tolerance pre-treated PD rats showed decreased intracranial LPS injection-induced dopaminergic neuronal loss in the SN

Unilateral striatal injection of $15 \mu \mathrm{g}$ LPS made a progressive and significant loss of the TH-positive neurons: $29.78 \%$ at 14 days, $54.59 \%$ at 28 days after the striatal
$15 \mu \mathrm{g}$ LPS exposure; however, no differences have been detected at 1 or 7 days, compared with the group receiving striatal $3 \mu \mathrm{l} \mathrm{PBS}$ exposure (Additional file 2: Figure S2).

To probe whether endotoxic tolerance pre-treated PD rats could decrease neuroinflammation-induced dopaminergic neuronal loss, we carried out immunohistochemical staining of $\mathrm{TH}$ and stereological counting of TH-positive neurons in the SN. As showed in Fig. 11, the striatal LPS injection decreased the total numbers of TH-positive neurons over time in both two groups; however, this reduction was more rapid and severe in group without endotoxic tolerance preconditioning. Peripheral LPS preconditioning made a $23.67 \%$ increase in the total numbers of TH-positive neurons (Fig. 11c). Moreover, the neuroprotection became more prominent at 28 days, with an increase of $54.64 \%$ (Fig. 11d).

The expression profiles of $\mathrm{TH}$ in the right $\mathrm{SN}$ were then detected by qRT-PCR. The TH mRNA level of PBS (i.p.) + LPS (striatum) group showed a more obvious decrease over time, with LPS (i.p.) + LPS (striatum) group as a control (Fig. 12a-c). At 14 days, the TH mRNA 


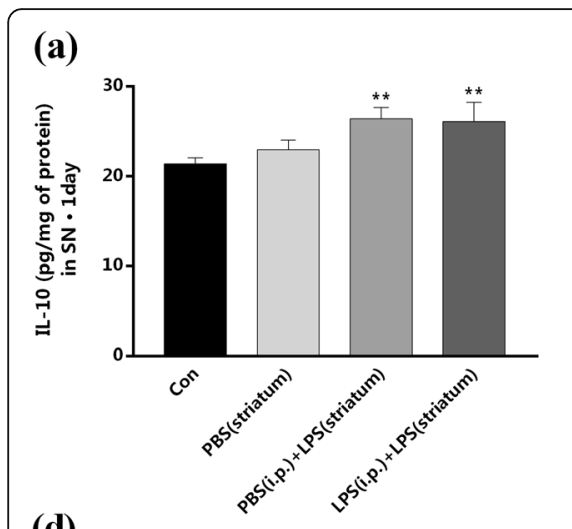

(d)

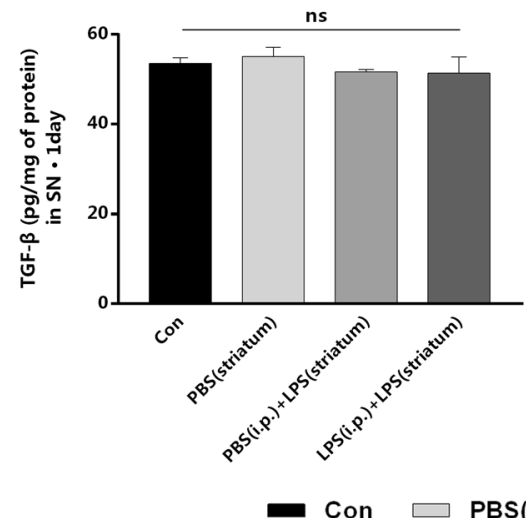

(e)

(b)
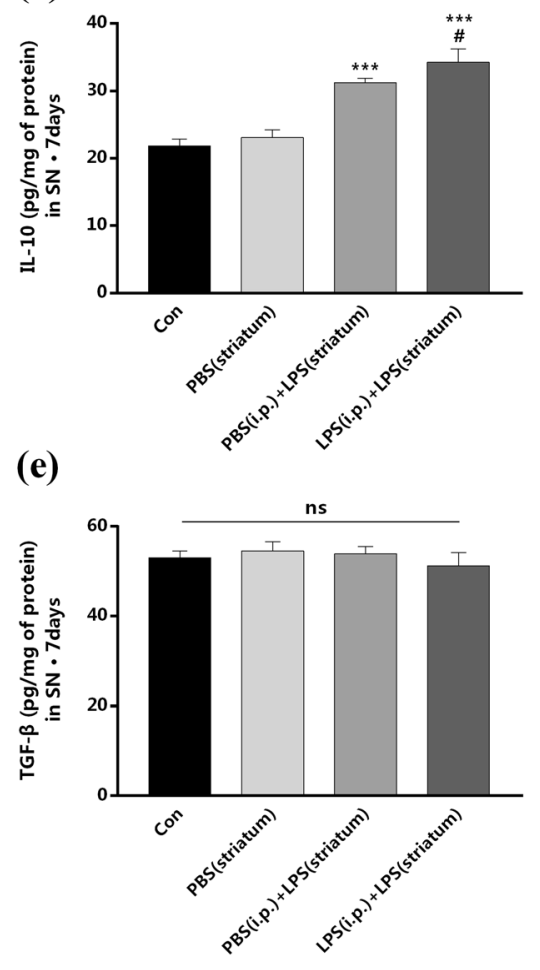

(f)
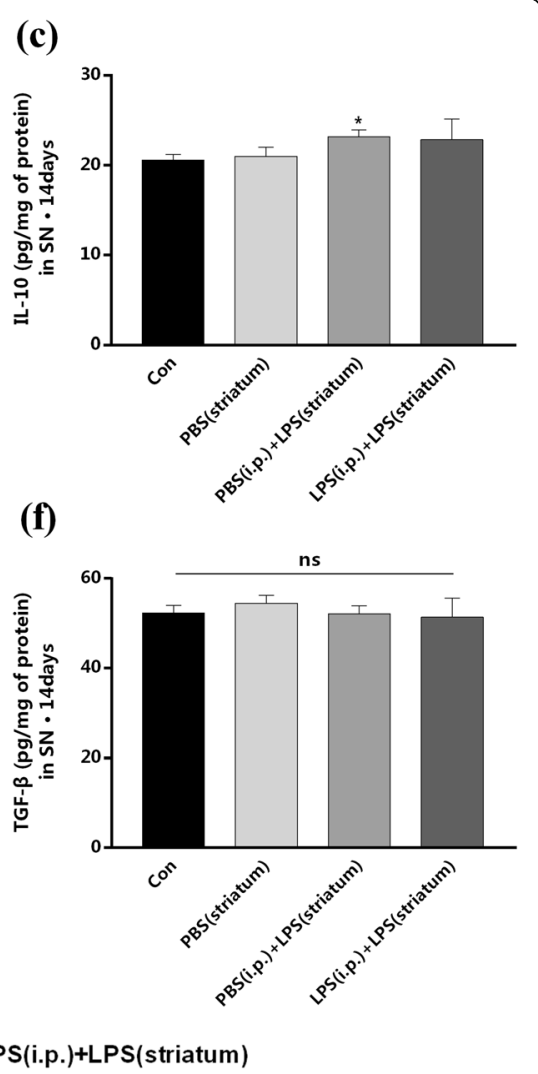

Fig. 7 Peripheral immune tolerance preconditioning did not alter intracranial LPS injection-induced production of IL-10 and TGF- $\beta$ in the SN. Rats from four groups with or without peripheral LPS pre-treatment were sacrificed, and right SN was dissected out at time point of 1, 7, 14, and 28 days after striatal LPS injection for ELISA analysis. The results indicated that the production of IL-10 was increased at 7 days after the intracranial LPS exposure, while there were no differences in our observation of IL-10 at any other time points as well as TGF- $\beta$ at any time points between the PBS (i.p.) + LPS (striatum) group and the LPS (i.p.) + LPS (striatum) group. a-f The expression of IL-10 (a-c) and TGF- $\beta$ (d-f in the right SN were detected by ELISA. ${ }^{*} P<0.05,{ }^{*} P<0.01,{ }^{* *} P<0.001$ vs. control group. $\# P<0.05$ vs. PBS (i.p.) + LPS (striatum) group. The data are presented as the mean $\pm \operatorname{SEM}(n=7)$

level in two groups received striatal LPS administration were 0.763 and 0.948 times of control group (Fig. 12b), it revealed that endotoxic tolerance pre-treatment upregulated the TH mRNA level $(P<0.01)$; moreover, the difference became more obvious at 28 days (Fig. 12c), while the difference was not shown at 7 days (Fig. 12a, b). Striatal vehicle injection [PBS (i.p.) group] made no differences in the $\mathrm{TH}$ expression compared with the control group.

These results underlined that endotoxic tolerance pretreatment could decrease intracranial LPS injectioninduced dopaminergic neuronal loss.

Peripheral immune tolerance pre-treated PD rats showed improved intracranial LPS injection-induced behavioral impairment

Test for amphetamine-induced rotational behavior was performed at 28 days post to the striatal LPS injection to assess the behavioral impairment. As shown in Fig. 13, striatal LPS injection caused a marked ipsilateral rotational behavior toward the lesion side in the PBS (i.p.) + LPS (striatum) group. A noticeable decrease (59.65\%, $P<0.01$ ) of the ipsilateral turns was developed by peripheral LPS pre-treated, compared with the PBS (i.p.) + PBS (striatum) group. Striatal vehicle injection [PBS (i.p.) group] made no differences in the behavior change at least 28 days compared with the control group.

These results clarified that endotoxic tolerance pretreatment could improve intracranial LPS injectioninduced behavioral impairment.

\section{Discussion}

Neuroinflammation has been established as a crucial mechanism in neurodegeneration. Moreover, a growing body of evidence demonstrates that periphery immunity exerts significant impact on neuroinflammation. Our recent study has identified the key roles of PBM and microglia in inflammatory transmission from the 


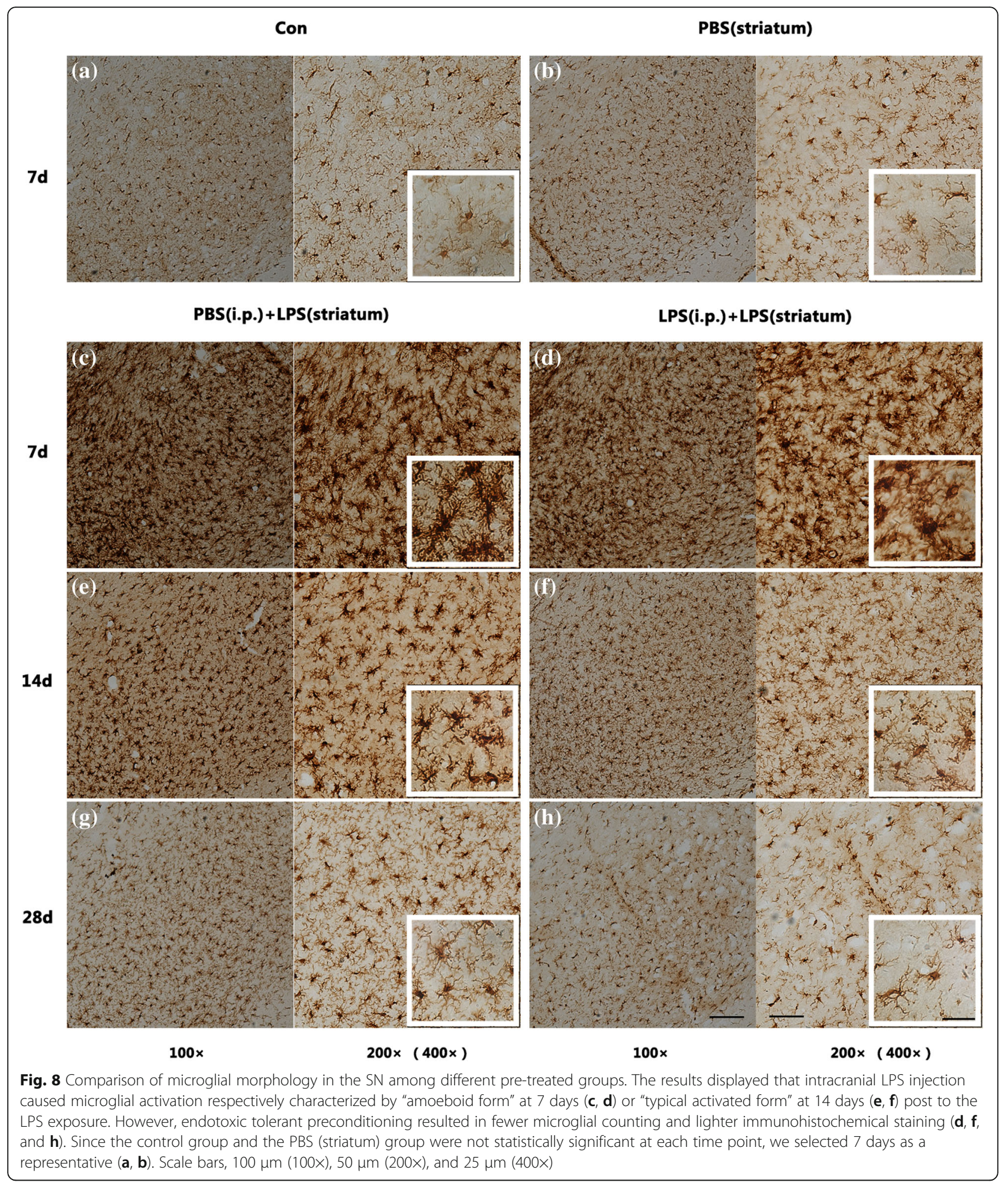

periphery to the brain, demonstrating the strong influence of flamed peripheral inflammation on brain immune reactivity [14]. Immune tolerance restricts excessive inflammation and serves as a vital mechanism to avoid unnecessary by-stander injury, while monocyte/macrophage serves as the main cells responsible for the induction of immune tolerance in vivo [28]. Here, we investigated the effects of peripheral immune tolerance pre-treatment on neuroinflammationinduced neurodegeneration. 


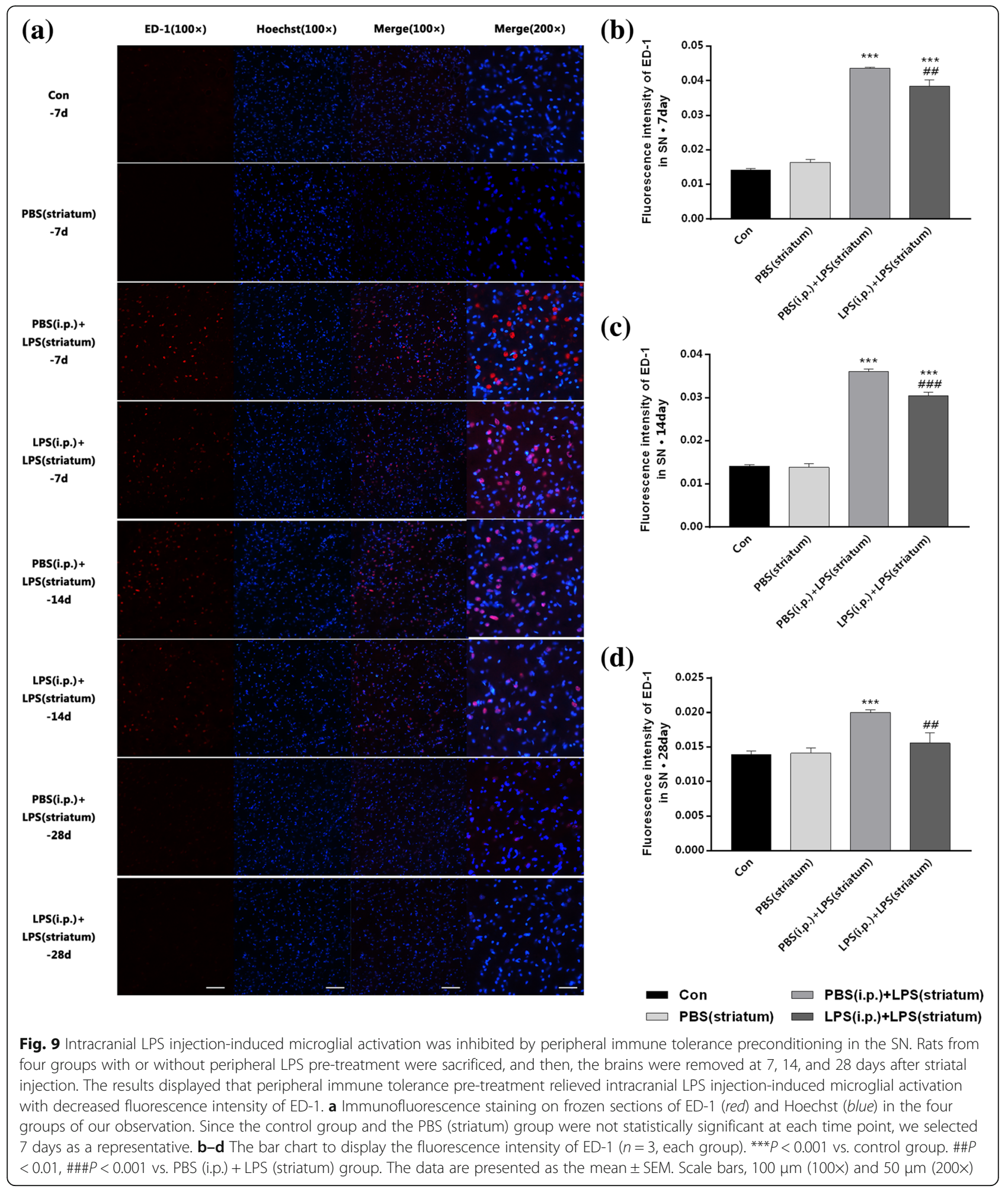

Peripheral immune tolerance suppresses inflammatory activity of peripheral blood monocyte

Immune tolerance is a protective mechanism facing to excessive inflammation and unnecessary by-stander injury [29]. Endotoxic tolerance is always induced by a low dose of LPS and deemed as the most essential component of immune tolerance when suffering from subsequent inflammatory challenge $[20,30]$. Our study indicated that repeated intraperitoneal injection of $0.3 \mathrm{mg} / \mathrm{kg}$ LPS for consecutive 4 days is indeed to induce 

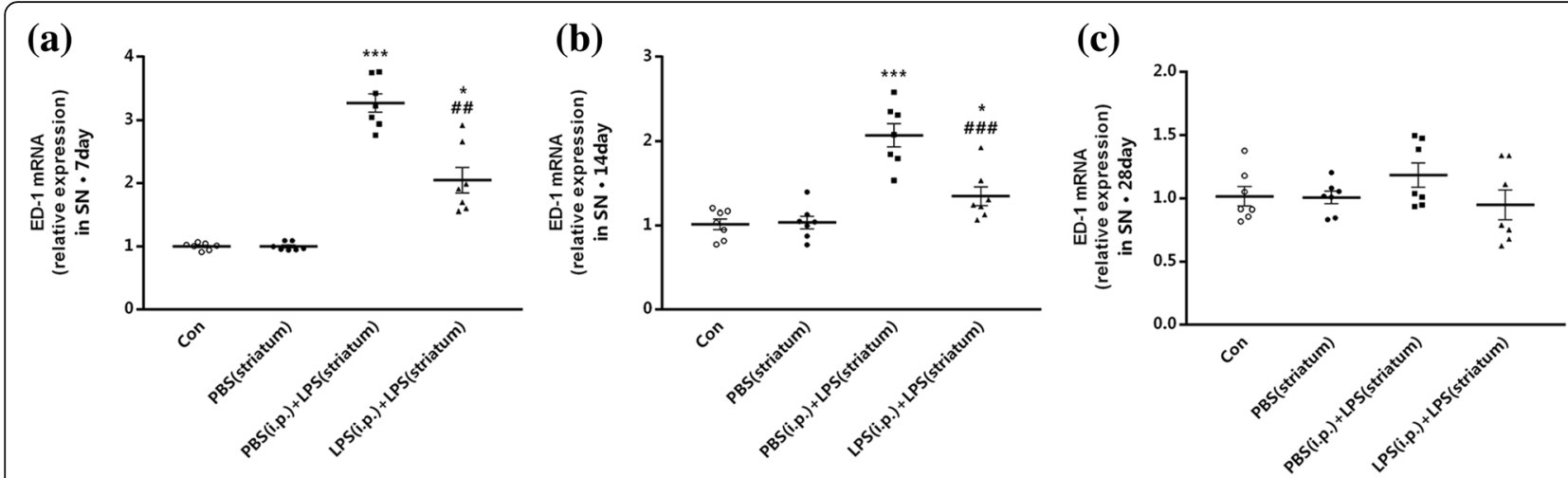

- Con - PBS(striatum) - PBS(i.p.)+LPS(striatum) - LPS(i.p.)+LPS(striatum)

Fig. 10 Intracranial LPS injection-induced microglial activation was inhibited by peripheral immune tolerance preconditioning in the SN. Rats from four groups with or without peripheral LPS pre-treatment were sacrificed, and then, the brains were removed at 7, 14, and 28 days after striatal injection. The results displayed that peripheral immune tolerance pre-treatment relieved intracranial LPS injection-induced microglial activation with downregulated mRNA levels of ED-1. a-c The mRNA levels of ED-1 were quantified by qRT-PCR ( $n=7$, each group). ${ }^{*} P<0.05,{ }^{* * *} P<0.001$ vs. control group. \#\#P<0.01, \#\#\#P<0.001 vs. PBS (i.p.) + LPS (striatum) group. The data are presented as the mean \pm SEM

the endotoxic tolerance of PBM characterized by decreased pro-inflammatory cytokines (TNF- $\alpha, \mathrm{IL}-1 \beta)$ and downregulated TLR4 expression under further LPS restimulation [31, 32]. Decreased inflammatory cytokines showed the peripheral immune tolerance inducedhypoactive state of PBM. TLR4 serve as the major pattern recognition receptors involved in the detection of LPS so that downregulation of TLR4 expression means diminished inflammatory response of PBM [33, 34]. This proves that $\mathrm{PBM}$ are the decisive cells in the formation of LPS-induced peripheral immune tolerance.

Repeated low-dose LPS injection is failing to cause neuroinflammation or dopaminergic neuronal loss

Our previous study has proved that the peripheral high-dose LPS $(5 \mathrm{mg} / \mathrm{kg}$ ) injection could induce an inflammatory effect in the $\mathrm{SN}$ as demonstrated by the increase of pro-inflammatory cytokines and microglial activation [14], which was in line with the classical study carried out by Qin et al. [6]. However, the dose of LPS we used in the present study was considered to be a low-dose $(0.3 \mathrm{mg} / \mathrm{kg})$. Intracephalic immune status was monitored after repeated low-dose LPS injection-induced peripheral immune tolerance. The results indicated that repeated low-dose LPS intraperitoneal injection was insufficient to cause inflammation within the SN of healthy rats (there was neither an increase of inflammatory cytokines nor an upregulation of Iba-1). Previous studies demonstrate that the levels and distribution of inflammatory factors in the brain vary with the various dosage of peripheral LPS administration [4, 35]. Quan et al. confirmed that high dose of LPS induced global expression of proinflammatory cytokines in the brain, whereas low dose of LPS increased TNF- $\alpha$ and IL-1 $\beta$ mRNA expression only in the choroid plexus, circumventricular organs, and meninges without brain parenchyma [36]. This conclusion coincides with our results, although other regions outside the $\mathrm{SN}$ have not been measured in our study. In addition, we also observed the neuronal morphology and the total numbers of dopaminergic neurons in the $\mathrm{SN}$ after repeated intraperitoneal LPS administration. Our results revealed that the immunoreactivity of $\mathrm{TH}$ expressed on dopaminergic neurons was preserved in the SN.

Peripheral immune tolerance pre-treatment alleviates the intracranial LPS injection-induced neuroinflammation

In the present study, we investigated the role of peripheral immune tolerance in the occurrence and progress of subsequent intracranial LPS injectionneuroinflammation. Our results showed that peripheral immune tolerance preconditioning attenuates the intracranial LPS injection-induced neuroinflammation, manifested as the decrease of inflammatory cytokines and the inhibition of microglial activation. This suggests that the regulation of $\mathrm{PBM}$ immune status could affect the progression of neuroinflammation [13]. We speculated that the neuroinflammatory alleviation was induced by peripheral immune tolerance preconditioning through the following ways: (a) Monocytes and microglia are considered to share the same origin of the myeloid lineage [37]; thus, immune tolerance pre-treatment induced hypo-responsiveness of monocytes may reflect the weakened immune activity of microglia in the brain correspondingly [38]. (b) Immune tolerant PBM may decrease the pro-inflammatory cytokines infiltrating from periphery into the brain correspondingly $[39,40]$. (c) Peripheral immune tolerance may impair immune activity of PBM, which would migrate from the periphery into the brain 


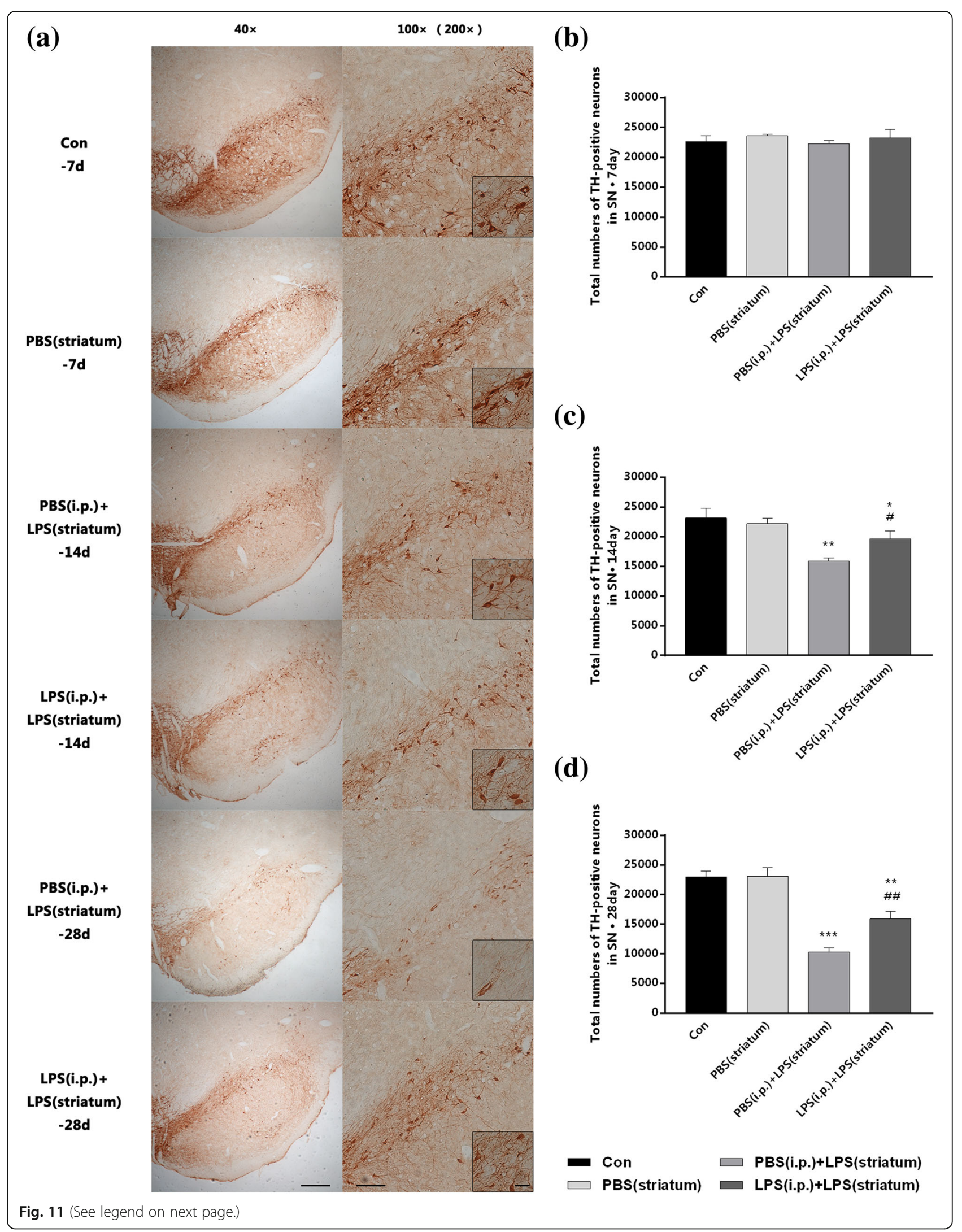


(See figure on previous page.)

Fig. 11 Peripheral immune tolerance pre-treated PD rats were protected from intracranial LPS injection-induced neurodegeneration with increased dopaminergic neuronal survival in the SN. Rats from four groups with or without peripheral LPS pre-treatment were sacrificed, and then, brains were removed at 7, 14, and 28 days after striatal injection. The results implied that peripheral immune tolerance pre-treatment protected the PD rats from neuroinflammation-related neurodegeneration with increased dopaminergic neuronal survival. a Immunohistochemistry staining on frozen sections of TH in the four groups of our observation. Since the control group and the PBS (striatum) group were not statistically significant at each time point, we selected 7 days as a representative. Moreover, the difference had not been distinguished as early as 7 days, so the data of the PBS (i.p.) + LPS (striatum) group and the LPS (i.p.) + LPS (striatum) group at 7 days was not shown. b-d Total numbers of THpositive neurons in the injected side of SN (right SN) collected by stereological counting $\left(n=3\right.$, each group). ${ }^{*} P<0.05$, ${ }^{* *} P<0.01,{ }^{* * *} P<0.001$ vs. control group. $\# P<0.05, \# \#>0.01$ vs. PBS (i.p.) + LPS (striatum) group. The data are presented as the mean \pm SEM. Scale bars, $250 \mu m(40 \times)$, $100 \mu \mathrm{m}(100 \times)$, and $25 \mu \mathrm{m}(200 x)$

through development into microglia directly or interacting with microglia indirectly to aggravate neuronal damage after neuroinflammation [41, 42]. (d) Peripheral immune tolerance may reshape the central microenvironment under neuroinflammation challenge by lessening interaction between PBM and other cells in the brain, such as perivascular cells [43] as well as endothelial cells of the BBB [44]. (e) Hypo-reactive PBM induced by peripheral immune tolerance may be associated with reduced $\mathrm{T}$ cellular infiltration into the brain $[17,45]$.

\section{Peripheral immune tolerance pre-treatment protects rats} from neuroinflammation-induced neurodegeneration

The incidence of peripheral immune tolerance has been reported in several systemic disease settings, including sepsis, trauma, surgery, and pancreatitis, underlining its clinical significance [30]. In this study, we evaluated the effect of peripheral immune tolerance on neuroinflammation-related neurodegeneration, and our results suggested that LPS preconditioning could protect rats from neuroinflammation-induced neurodegeneration, showing as the increase of neuronal survival and the improvement of behavioral damage. Rosenzweig et al. clarified that LPS preconditioning modulates the cellular inflammatory response after cerebral ischemia, resulting in neuroprotection [21]. Besides, LPS preconditioning conferred neuroprotection has been proved in traumatic brain injury [22] and deep hypothermic circulatory arrest [23]. In accordance with these results, we observed that peripheral immune tolerant preconditioning-induced hypo-responsiveness of PBM led to a significant neuroprotection from subsequent neurodegeneration. It is sufficient to show that PBM serve as a significant regulator in the development and progression of neurodegenerative diseases.

It is recognized that excessive microglial activation can lead to the development of neurodegenerative diseases [9]; on the contrary, our present study showed that the neuroprotection of LPS preconditioning from neurodegeneration was accompanied by inhibition of microglial activation. Rodents that pre-treated with fluoxetine or somatostatin showed neuroprotective effects depending on inhibition of neuroinflammation-related microglial

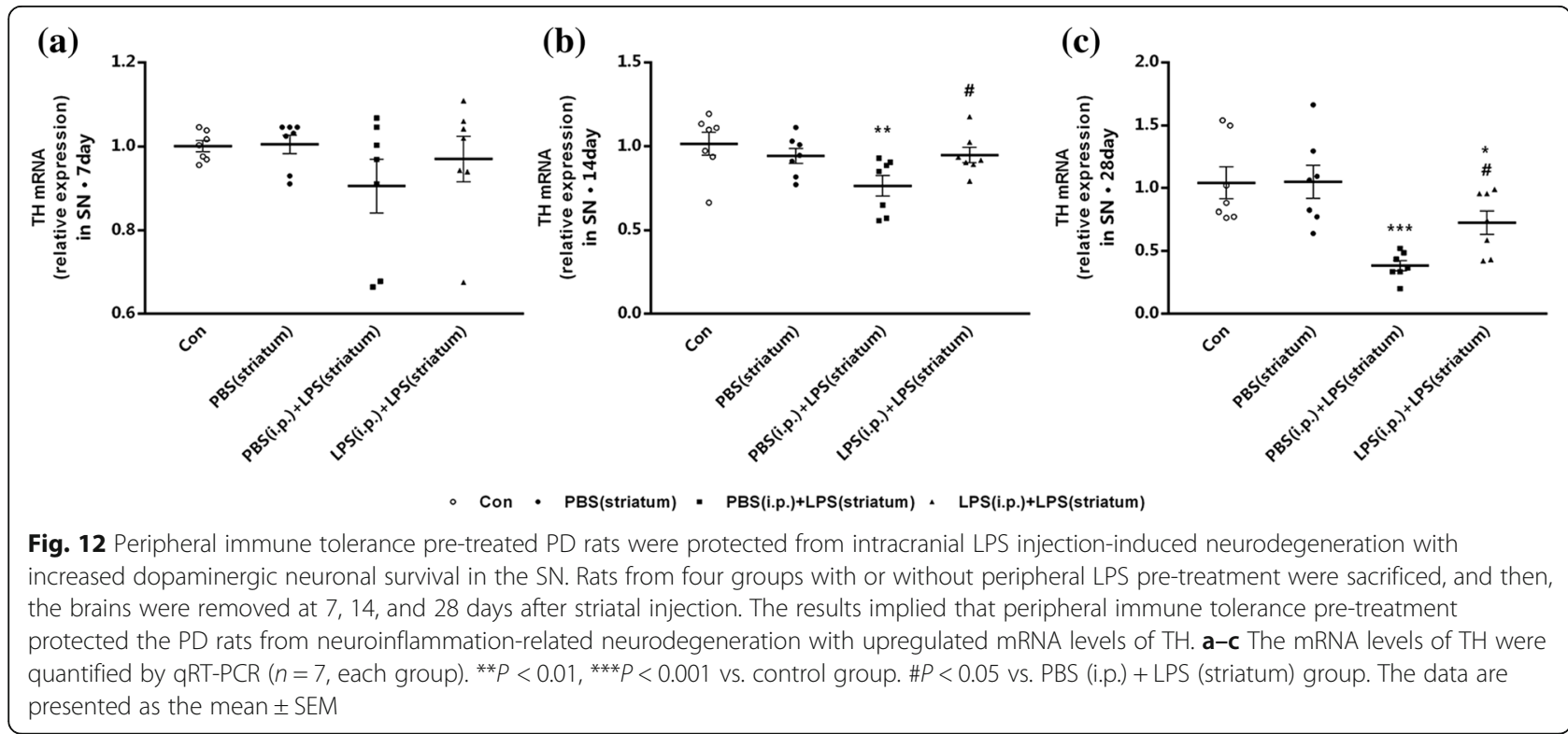




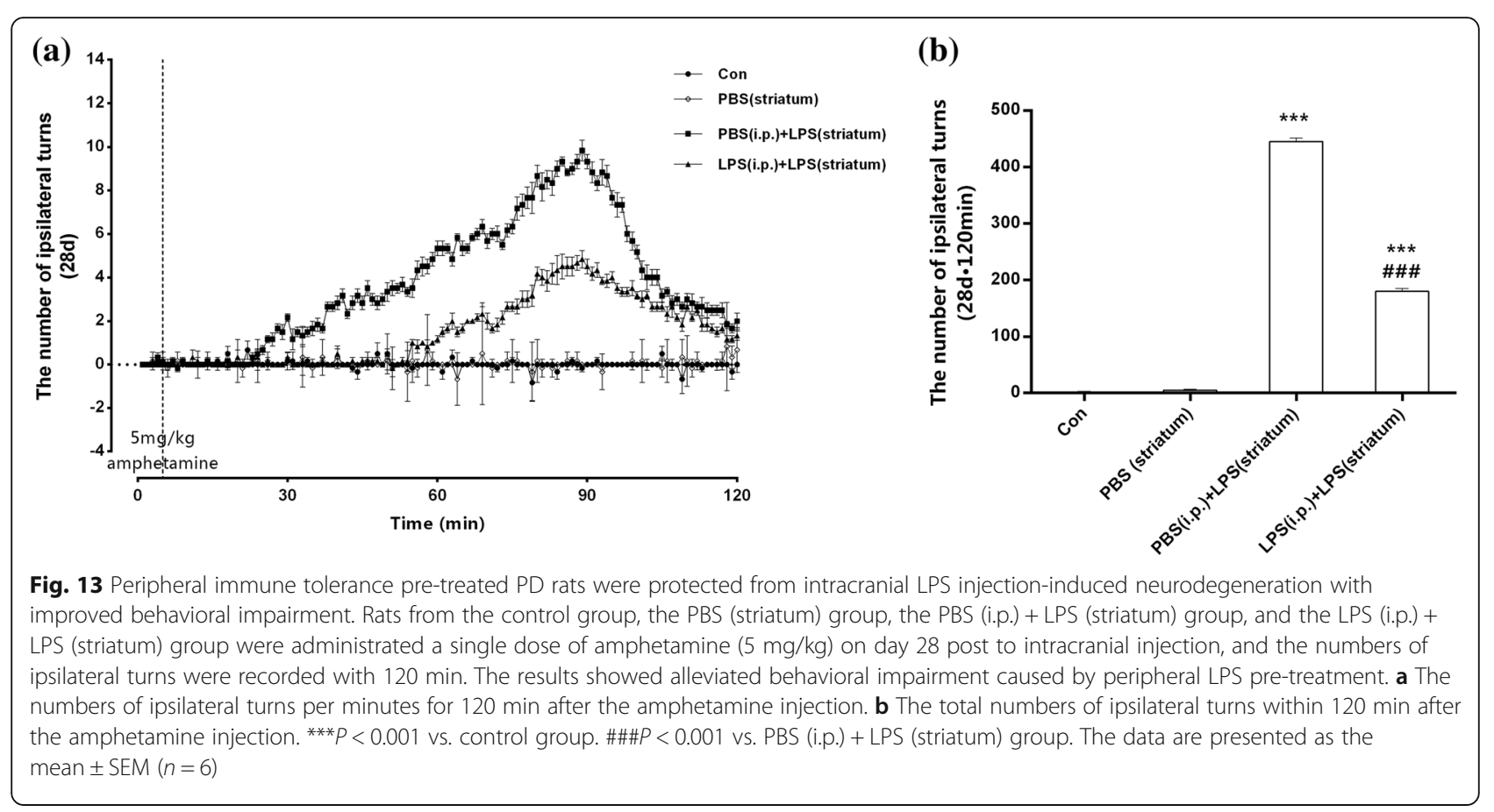

activation [46, 47]. These results highlight the neuroprotective role of microglial immunocompetent restriction in neuroinflammation-induced neurodegenerative diseases. Therefore, inhibition of microglial over-activation caused by peripheral immune tolerance produces neuroprotection.

Taken together, our study provides evidence that peripheral immune tolerance preconditioning play a crucial role in neuroprotection from subsequent neuroinflammationinduced neurodegeneration, and both PBM and microglia may serve as potential significant elements in this neuroprotection. Being different from previous studies, we regarded neurodegeneration as systemic diseases rather than disorders limited in the CNS. The results of our study may provide evidences for the immunologic communication between brain and periphery and likely produce an easier and more convenient peripheral way to manipulate the immunologic activities within the brain. However, our study is limited in that the study of molecular mechanisms and signaling pathways under this neuroprotection needs to be further implemented.

\section{Conclusions}

Peripheral immune tolerance is an important regulatory mechanism for the immunological activity of PBM. Our results strongly indicated that peripheral immune tolerance attenuated subsequent neuroinflammation and provided neuroprotection from neuroinflammationinduced neurotoxicity, which will provide new therapeutic approaches for neurodegenerative diseases.

\section{Additional files}

Additional file 1: Figure S1. Repeated intraperitoneal injection of $0.3 \mathrm{mg} / \mathrm{kg}$ LPS for 4 days induced peripheral immune tolerance of PBM. Peripheral blood monocytes of rats from the five groups pre-treated with PBS $(0.3 \mathrm{ml} / \mathrm{kg})$, LPS $(0.1,0.3$, or $0.9 \mathrm{mg} / \mathrm{kg})$ or without pre-treatment (control group) were isolated and cultured, and then, monocytes were restimulated by a single high-dose LPS (100 ng) for $4 \mathrm{~h}$ in vitro. The production of TNF-a and the expression of TLR4 in PBM were downregulated after LPS restimulation by repeated $0.3 \mathrm{mg} / \mathrm{kg}$ LPS intraperitoneal injections in $0.3 \mathrm{mg} / \mathrm{kg}$ LPS (i.p.) group, while the similar downregulation of inflammation was not observed in the other two groups with repeated 0.1 or $0.9 \mathrm{mg} / \mathrm{kg}$ LPS intraperitoneal injection. ( $a-b)$ The levels of TNF- $a(a)$ and $I L-1 \beta(b)$ in the supernatant were detected by ELISA. (c-d) TLR4 production in PBM was quantified by measuring band intensities using ImageJ software. The values were normalized to GAPDH. ${ }^{* *} P<0.01$, ${ }^{* * *} P<0.001$ vs. control group. $\# P<0.05$, \#\#P<0.01, \#\#\#P<0.001 vs. PBS (i.p.) group. Data are presented as the mean \pm SEM $(n=6)$. (TIFF $275 \mathrm{~kb})$

Additional file 2: Figure S2. Striatal injection of $15 \mu \mathrm{g}$ LPS-induced generation and loss of dopaminergic neurons in the SN. A single dose of vehicle ( $5 \mu \mathrm{l}$ PBS) or LPS (15 $\mu \mathrm{g}$ in $5 \mu \mathrm{l}$ PBS) was administered to the right striatum in the PBS (striatum) group or in the LPS (striatum) group individually, and there was a control group without any treatment. The brains were removed at time point of 1, 7, 14, and 28 days after striatal injection, and the right SN was dissected out. The results implied that striatal

injection of $15 \mu \mathrm{g}$ LPS induced significant loss of dopaminergic neurons in the SN. (a) Immunohistochemistry staining on frozen sections of $\mathrm{TH}$ in the three groups of our observation. Since the control group and the PBS (striatum) group were not statistically significant at each time point, we selected control group as a representative. Moreover, the difference

had not been distinguished as early as 7 days, so the data of the PBS (striatum) group and the LPS (striatum) group at 1 and 7 days was not shown. (b) Total numbers of TH-positive neurons in the injected side of SN (right SN) collected by stereological counting. \#\#P<0.01 vs. PBS (striatum) group. Data are presented as the mean \pm SEM $(n=3)$. Scale bars, $250 \mu \mathrm{m}$ (40x), $100 \mu \mathrm{m}$ (100x), and $25 \mu \mathrm{m}$ (200x). (TIFF $3252 \mathrm{~kb}$ ). 


\section{Abbreviations}

ANOVA: One-way analysis of variance; BBB: Blood-brain barrier; CNS: Central nervous system; ELISA: Enzyme-linked immunosorbent assay; ET: Endotoxic tolerance; i.p.: Intraperitoneal; IL-10: Interleukin-10; IL-1B: Interleukin-1 $\beta$; LPS: Lipopolysaccharide; LSD: Least Significant Digit; PBM: Peripheral blood monocytes; PBS: Phosphate buffered saline; PD: Parkinson's disease; PVDF: Polyvinylidene difluoride membranes; SEM: Standard error of the mean; SN: Substantia nigra; TGF- $\beta$ : Transforming growth factor- $\beta$; TLR4: Tolllike receptor 4; TNF-a: Tumor necrosis factor-a

\section{Acknowledgements}

Not applicable.

\section{Funding}

This research was supported by the National Nature Science Foundation of China (grant no. 81371421).

\section{Availability of data and materials}

Data supporting the conclusions of this article are presented in the manuscript.

\section{Authors' contributions}

$X-G L$ and $Y L$ conceived and designed the experiments. YL, XX, L-PX, HL, and FL performed the experiments. YL, XX, L-PX, YR, and Z-YH analyzed the data, and $Y L$ and $X-G L$ wrote the paper. All authors read and approved the final manuscript.

\section{Ethics approval}

All animal procedures performed in this study were reviewed and approved by the Animal Center of China Medical University and carried out according to the National Institute of Health Guidelines (National Institutes of Health, Bethesda, USA).

\section{Consent for publication}

Not applicable.

\section{Competing interests}

The authors declare that they have no competing interests.

\section{Publisher's Note}

Springer Nature remains neutral with regard to jurisdictional claims in published maps and institutional affiliations.

Received: 12 July 2017 Accepted: 2 November 2017 Published online: 16 November 2017

\section{References}

1. Ghadery C, Koshimori Y, Coakeley S, Harris M, Rusjan P, Kim J, et al. Microglial activation in Parkinson's disease using [18F]-FEPPA. J Neuroinflammation. 2017;14(1):8-16. http://www.ncbi.nlm.nih.gov/pubmed/ 28086916 .

2. Heneka MT, Carson MJ, El Khoury J, Landreth GE, Brosseron F, Feinstein DL, et al. Neuroinflammation in Alzheimer's disease. Lancet Neurol. 2015;14(4): 388-405. http://www.ncbi.nlm.nih.gov/pubmed/25792098.

3. Pott Godoy MC, Tarelli R, Ferrari CC, Sarchi MI, Pitossi FJ. Central and systemic IL-1 exacerbates neurodegeneration and motor symptoms in a model of Parkinson's disease. Brain. 2008;131:1880-94. http://www.ncbi.nlm. nih.gov/pubmed/18504291.

4. Qin XY, Zhang SP, Cao C, Loh YP, Cheng Y. Aberrations in peripheral inflammatory cytokine levels in Parkinson disease: a systematic review and meta-analysis. JAMA Neurol. 2016;73(11):1316-24. http://www.ncbi.nlm.nih. gov/pubmed/27668667

5. Machado A, Herrera AJ, Venero JL, Santiago M, De Pablos RM, Villaran RF, et al. Peripheral inflammation increases the damage in animal models of nigrostriatal dopaminergic neurodegeneration: possible implication in Parkinson's disease incidence. Parkinsons Dis. 2011;2011:393769. http://www. ncbi.n/m.nih.gov/pubmed/21603178 .

6. Qin L, Wu X, Block ML, Liu Y, Breese GR, Hong JS, et al. Systemic LPS causes chronic neuroinflammation and progressive neurodegeneration. Glia. 2007; 55(5):453-62. http://www.ncbi.nlm.nih.gov/pubmed/17203472 .
7. Driver JA, Logroscino G, Lu L, Gaziano JM, Kurth T. Use of non-steroidal antiinflammatory drugs and risk of Parkinson's disease: nested case-control study. BMJ. 2011;342:d198. http://www.ncbi.nlm.nih.gov/pubmed/21252104 .

8. Sung YF, Liu FC, Lin CC, Lee JT, Yang FC, Chou YC, et al. Reduced risk of Parkinson disease in patients with rheumatoid arthritis: a nationwide population-based study. Mayo Clin Proc. 2016;91 (10):1346-53. http://www. ncbi.nlm.nih.gov/pubmed/27712633

9. Colonna M, Butovsky O. Microglia function in the central nervous system during health and neurodegeneration. Annu Rev Immunol. 2017;35:441-68. http://www.ncbi.nlm.nih.gov/pubmed/28226226.

10. Luo XG, Chen SD. The changing phenotype of microglia from homeostasis to disease. Transl Neurodegener. 2012;1:9-21. http://www.ncbi.nlm.nih.gov/ pubmed/23210447

11. Fang M, Yuan Y, Lu J, Li HE, Zhao M, Ling EA, et al. Scutellarin promotes microglia-mediated astrogliosis coupled with improved behavioral function in cerebral ischemia. Neurochem Int. 2016;97:154-71. http://www.ncbi.nIm. nih.gov/pubmed/27105682

12. Tian L, Rauvala H, Gahmberg CG. Neuronal regulation of immune responses in the central nervous system. Trends Immunol. 2009:30(2):91-9. http:// www.ncbi.nlm.nih.gov/pubmed/19144568 .

13. Minogue AM. Role of infiltrating monocytes/macrophages in acute and chronic neuroinflammation: effects on cognition, learning and affective behaviour. Prog Neuro-Psychopharmacol Biol Psychiatry. 2017;79:15-8. http://www.ncbi.nlm.nih.gov/pubmed/28189704

14. Xie X, Luo X, Liu N, Li X, Lou F, Zheng Y, et al. Monocytes, microglia, and CD200-CD200R1 signaling are essential in the transmission of inflammation from the periphery to the central nervous system. J Neurochem. 2017; 141(2):222-35. http://www.ncbi.nlm.nih.gov/pubmed/28164283

15. Prinz M, Erny D, Hagemeyer N. Ontogeny and homeostasis of CNS myeloid cells. Nat Immunol. 2017;18(4):385-92. http://www.ncbi.nlm.nih.gov/ pubmed/28323268

16. Gomez Perdiguero E, Klapproth K, Schulz C, Busch K, Azzoni E, Crozet L, et al. Tissue-resident macrophages originate from yolk-sac-derived erythromyeloid progenitors. Nature. 2015;518(7540):547-51. http://www.ncbi.nlm. nih.gov/pubmed/25470051

17. Waschbisch A, Schroder S, Schraudner D, Sammet L, Weksler B, Melms A, et al. Pivotal role for CD16+ monocytes in immune surveillance of the central nervous system. J Immunol. 2016;196(4):1558-67. http://www.ncbi.nlm.nih. gov/pubmed/26746191

18. Burdo TH, Lackner A, Williams KC. Monocyte/macrophages and their role in HIV neuropathogenesis. Immunol Rev. 2013;254(1):102-13. http://www.ncbi. nlm.nih.gov/pubmed/23772617.

19. Kox M, de Kleijn S, Pompe JC, Ramakers BP, Netea MG, van der Hoeven JG, et al. Differential ex vivo and in vivo endotoxin tolerance kinetics following human endotoxemia. Crit Care Med. 2011;39(8):1866-70. http://www.ncbi. nlm.nih.gov/pubmed/21494102 .

20. Martin M, Katz J, Vogel SN, Michalek SM. Differential induction of endotoxin tolerance by lipopolysaccharides derived from Porphyromonas gingivalis and Escherichia coli. J Immunol. 2001;167(9):5278-85. http://www.ncbi.nlm. nih.gov/pubmed/11673543.

21. Rosenzweig HL, Lessov NS, Henshall DC, Minami M, Simon RP, StenzelPoore MP. Endotoxin preconditioning prevents cellular inflammatory response during ischemic neuroprotection in mice. Stroke. 2004;35(11): 2576-81. http:/www.ncbinlm.nih.gov/pubmed/15375302.

22. Longhi L, Gesuete R, Perego C, Ortolano F, Sacchi N, Villa P, et al. Longlasting protection in brain trauma by endotoxin preconditioning. J Cereb Blood Flow Metab. 2011;31(2):1919-29. http:/www.ncbi.nlm.nih.gov/ pubmed/21468087

23. Hickey EJ, You X, Kaimaktchiev V, Stenzel-Poore M, Ungerleider RM. Lipopolysaccharide preconditioning induces robust protection against brain injury resulting from deep hypothermic circulatory arrest. J Thorac Cardiovasc Surg. 2007;133(6):1588-96. http://www.ncbi.nlm.nih.gov/ pubmed/17532961

24. Paxinos $G$, Watson CR, Emson PC. AChE-stained horizontal sections of the rat brain in stereotaxic coordinates. J Neurosci Methods. 1980;3(2):129-49. http://www.ncbi.nlm.nih.gov/pubmed/6110810

25. Kirik D, Rosenblad C, Bjorklund A, Mandel RJ. Long-term rAAV-mediated gene transfer of GDNF in the rat Parkinson's model: intrastriatal but not intranigral transduction promotes functional regeneration in the lesioned nigrostriatal system. J Neurosci. 2000;20(12):4686-700. http://www.ncbi.nlm. nih.gov/pubmed/10844038 
26. Kirik D, Rosenblad C, Bjorklund A. Characterization of behavioral and neurodegenerative changes following partial lesions of the nigrostriatal dopamine system induced by intrastriatal 6-hydroxydopamine in the rat. Exp Neurol. 1998;152(2):259-77. http://www.ncbi.n/m.nih.gov/pubmed/ 9710526

27. West MJ, Slomianka L, Gundersen HJ. Unbiased stereological estimation of the total number of neurons in the subdivisions of the rat hippocampus using the optical fractionator. Anat Rec. 1991;231(4):482-97. http://www. ncbi.nlm.nih.gov/pubmed/1793176 .

28. Lopez-Collazo E, del Fresno C. Pathophysiology of endotoxin tolerance: mechanisms and clinical consequences. Crit Care. 2013;17(6):242-52. http:// www.ncbi.nlm.nih.gov/pubmed/24229432 .

29. Seeley JJ, Ghosh S. Molecular mechanisms of innate memory and tolerance to LPS. J Leukoc Biol. 2017;101(1):107-19. http://www.ncbi.nlm.nih.gov/ pubmed/27780875

30. Biswas SK, Lopez-Collazo E. Endotoxin tolerance: new mechanisms, molecules and clinical significance. Trends Immunol. 2009;30(10):475-87. http://www.ncbi.nlm.nih.gov/pubmed/19781994

31. Alves-Rosa F, Vulcano M, Beigier-Bompadre M, Fernandez G, Palermo M, Isturiz MA. Interleukin-1beta induces in vivo tolerance to lipopolysaccharide in mice. Clin Exp Immunol. 2002;128(2):221-8. http://www.ncbi.nlm.nih.gov/ pubmed/12041508

32. Collins PE, Carmody RJ. The regulation of endotoxin tolerance and its impact on macrophage activation. Crit Rev Immunol. 2015;35(4):293-323. http://www.ncbi.nlm.nih.gov/pubmed/26757393.

33. Kim SJ, Kim HM. Dynamic lipopolysaccharide transfer cascade to TLR4/MD2 complex via LBP and CD14. BMB Rep. 2017;50(1):55-7. http://www.ncbi.nlm. nih.gov/pubmed/28115037.

34. Li J, Csakai A, Jin J, Zhang F, Yin H. Therapeutic developments targeting tolllike Receptor-4-mediated neuroinflammation. ChemMedChem. 2016;11(2): 154-65. http://www.ncbi.nlm.nih.gov/pubmed/26136385

35. Quan N, Whiteside M, Herkenham M. Time course and localization patterns of interleukin-1 $\beta$ messenger RNA expression in brain and pituitary after peripheral administration of lipopolysaccharide. Neuroscience. 1998;83(1): 281-93. http://www.ncbi.nlm.nih.gov/pubmed/9466417.

36. Quan N, Stern EL, Whiteside MB, Herkenham M. Induction of proinflammatory cytokine mRNAs in the brain after peripheral injection of subseptic doses of lipopolysaccharide in the rat. J Neuroimmunol. 1999;93: 72-80. http://www.ncbi.nlm.nih.gov/pubmed/10378870

37. Templeton SP, Kim TS, O'Malley K, Perlman S. Maturation and localization of macrophages and microglia during infection with a neurotropic murine coronavirus. Brain Pathol. 2008;18(1):40-51. http://www.ncbi.nlm.nih.gov/ pubmed/17935605

38. Reader BF, Jarrett BL, McKim DB, Wohleb ES, Godbout JP, Sheridan JF. Peripheral and central effects of repeated social defeat stress: monocyte trafficking, microglial activation, and anxiety. Neuroscience. 2015;289:42942. http://www.ncbi.nlm.nih.gov/pubmed/25596319.

39. Teeling $\mathrm{L}$, Perry VH. Systemic infection and inflammation in acute CNS injury and chronic neurodegeneration: underlying mechanisms. Neuroscience. 2009;158(3):1062-73. http://www.ncbi.nlm.nih.gov/pubmed/ 18706982 .

40. Turrin NP, Rivest S. Unraveling the molecular details involved in the intimate link between the immune and neuroendocrine systems. Exp Biol Med. 2004; 229(10):996-1006. http://www.ncbi.nlm.nih.gov/pubmed/15522835 .

41. Ruitenberg MJ, Wells J, Bartlett PF, Harvey AR, Vukovic J. Enrichment increases hippocampal neurogenesis independent of blood monocytederived microglia presence following high-dose total body irradiation. Brain Res Bull. 2017;132:150-9. http://www.ncbi.nlm.nih.gov/pubmed/28552674 .

42. Paouri E, Tzara O, Kartalou Gl, Zenelak S, Georgopoulos S. Peripheral Tumor Necrosis Factor-alpha (TNF-a) modulates amyloid pathology by regulating blood-derived immune cells and glial response in the brain of AD/TNF transgenic mice. J Neurosci. 2017;37(20):5155-71. http://www.ncbi.nlm.nih. gov/pubmed/28442538

43. Prodeus A, Cydzik M, Abdul-Wahid A, Huang E, Khatri I, Gorczynski R, et al. Agonistic CD200R1 DNA aptamers are potent immunosuppressants that prolong allogeneic skin graft survival. Mol Ther Nucleic Acids. 2014;3(3):1909. http://www.ncbi.nlm.nih.gov/pubmed/25158092 .

44. Man S, Tucky B, Cotleur A, Drazba J, Takeshita Y, Ransohoff RM. CXCL12induced monocyte-endothelial interactions promote lymphocyte transmigration across an in vitro blood-brain barrier. Sci Transl Med. 2012;4: 119-32. http://www.ncbi.nlm.nih.gov/pubmed/22301555 .
45. Gonzalez H, Contreras F, Pacheco R. Regulation of the neurodegenerative process associated to Parkinson's disease by CD4+ T cells. J Neuroimmune Pharmacol. 2015;10(4):561-75. http://www.ncbi.nlm.nih.gov/pubmed/ 26018603.

46. Chung ES, Chung YC, Bok E, Baik HH, Park ES, Park JY, et al. Fluoxetine prevents LPS-induced degeneration of nigral dopaminergic neurons by inhibiting microglia-mediated oxidative stress. Brain Res. 2010;1363:143-50. http://www.ncbi.nlm.nih.gov/pubmed/20858471 .

47. Bai L, Zhang X, Li X, Liu N, Lou F, Ma H, et al. Somatostatin prevents lipopolysaccharide-induced neurodegeneration in the rat substantia nigra by inhibiting the activation of microglia. Mol Med Rep. 2015;12(1):1002-8. http://www.ncbi.nlm.nih.gov/pubmed/25777539 .

\section{Submit your next manuscript to BioMed Central and we will help you at every step:}

- We accept pre-submission inquiries

- Our selector tool helps you to find the most relevant journal

- We provide round the clock customer support

- Convenient online submission

- Thorough peer review

- Inclusion in PubMed and all major indexing services

- Maximum visibility for your research

Submit your manuscript at www.biomedcentral.com/submit
Biomed Central 